\title{
LARGE DEVIATIONS PRINCIPLE FOR OCCUPANCY PROBLEMS WITH COLORED BALLS
}

\author{
PAUL DUPUIS, ${ }^{* *}$ Brown University \\ CARL NUZMAN $* * * *$ AND \\ PHIL WHITING, ${ }^{* * * * * * * *}$ Alcatel-Lucent
}

\begin{abstract}
A large deviations principle (LDP), demonstrated for occupancy problems with indistinguishable balls, is generalized to the case in which balls are distinguished by a finite number of colors. The colors of the balls are chosen independently from the occupancy process itself. There are $r$ balls thrown into $n$ urns with the probability of a ball entering a given urn being $1 / n$ (i.e. Maxwell-Boltzmann statistics). The LDP applies with the scale parameter, $n$, tending to infinity and $r$ increasing proportionally. The LDP holds under mild restrictions, the key one being that the coloring process by itself satisfies an LDP. This includes the important special cases of deterministic coloring patterns and colors chosen with fixed probabilities independently for each ball.
\end{abstract}

Keywords: Occupancy model; coloring process; Maxwell-Boltzmann statistics; sample path large deviations principle; circuit-switched network; population estimation; optical packet switching

2000 Mathematics Subject Classification: Primary 60F10

Secondary $05 \mathrm{~A} 16$

\section{Introduction}

In occupancy models which follow Maxwell-Boltzmann statistics, balls are thrown into $n$ urns with the probability of a ball entering a given urn being $1 / n$, independent of all other balls. References [2] and [7] developed sample path large deviations principles for scaled occupancy processes in which the time variable is (approximately) the number of balls thrown per urn, the state is given by the fraction of urns which contain exactly $i$ balls, and the number of balls and urns are scaled up in fixed proportion. An LDP was obtained for infinite-dimensional processes in [2], whilst [7] focused on processes with a finite number of occupancy levels, $i=0, \ldots, I$, and with $i=I+$ for urns with more than $I$ balls. Additionally, [7] provided explicit solutions to the corresponding calculus of variations problem.

In this paper we consider a generalization of such occupancy models to allow balls with more than one color. We fix on the case of two colors, as the extension to any finite number of colors is straightforward. The overall process can be regarded as the conjunction of two independent random processes: an occupancy process which determines which urn each ball

\footnotetext{
Received 26 November 2003; revision received 23 May 2005.

* Research supported in part by the National Science Foundation (NSF-DMS-0306070 and NSF-DMS-0404806) and the Army Research Office (DAAD19-02-1-0425).

** Postal address: Lefschetz Center for Dynamical Systems, Division of Applied Mathematics, Brown University, 182 George Street, Providence, RI 02912, USA.

*** Postal address: Bell Labs, Alcatel-Lucent, 600 Mountain Avenue, Murray Hill, NJ 07974, USA.

**** Email address: pwhiting@ research.bell-labs.com
} 
enters and a second process that determines color. The coloring process can be quite general and includes the important special case where each color is picked independently and according to a fixed vector of probabilities (independent and identically distributed (i.i.d.) coloring), as well as deterministic coloring patterns. Again, time is scaled by a factor of $n$, so that at time $t$, $t \approx k / n \in[0, \tau], k$ balls have been thrown. The state of the process is the empirical measure, which records the fraction of urns that contain $i$ balls of color 1 and $j$ balls of color 2 , for $0 \leq i \leq I+$ and $0 \leq j \leq J+$, where $I+$ and $J+$ correspond to more than $I$ balls and more than $J$ balls, respectively. Thus, $\Gamma_{i, j}^{n}(t)$ is the fraction of urns containing $i$ color- 1 balls and $j$ color- 2 balls after approximately $n t$ balls have been thrown. In general, the process $\left\{\Gamma_{i, j}^{n}(t)\right\}$ is not Markov unless the coloring process is i.i.d.

There is a wide literature on occupancy problems, and the case of distinguished classes of balls is a common generalization [11], [13]. At the end of this section, we describe a number of applied problems in which colored balls play an important role. In part, our interest in large deviation approximations is motivated by the fact that occupancy models are one of the very few instances where a large deviations analysis can actually be fully exploited. In the great majority of potential applications of large deviations, the final result is a variational characterization of some important performance measure or other quantity. As a practical matter, in all but the simplest settings the solution of these variational problems is not currently within our computational reach. In contrast, for a number of single-color occupancy models the potential of the theory can be realized, in that the variational problem can be solved explicitly. The class of 'solvable' problems has always played the central role in practical applications of mathematics, and is frequently turned to even when the underlying assumptions are far from satisfied.

It is thus of some interest to understand the degree to which 'solvability' is generic in occupancy models. In the case of problems with color, several new phenomena appear which may have an impact on the issue of explicit solutions. In the case of a single color, the components of the minimizing trajectories have a one-dimensional ordered dependence, and the form of the component with a given coordinate index is determined by the component with index one lower. In contrast, we will see that for the case of color there is a multi-dimensional dependence. Another important distinction is that, while occupancy models with a single color have a process level rate function that is convex, this is no longer true for the multi-color case if there is sufficient correlation in the coloring process (see the examples in Section 2 and the discussion in Section 4). The impact of both these effects on the issue of explicit solutions is a topic for further investigation.

We derive the LDP for the color occupancy processes by using the representation theorem for the scaled log-moment generating functions for measurable functions of sample paths (see [4]). The representation is as an infimum over measures of the sum of a relative entropy cost and a terminal cost. As discussed in Section 2, there is a natural split of the relative entropy cost between a cost for occupancy and one for the coloring process. The local rate function in the single-color case is the relative entropy $R(\theta(t) \| \gamma(t))$, where $\gamma_{i}(t)$ is interpreted as the asymptotic proportion of urns that contain $i$ balls and $\theta_{i}(t)$ as the rate at which balls enter such urns when the time is $t$ [7]. The corresponding expression in the color case is a weighted sum of relative entropy terms

$$
\dot{x}_{1}(t) R\left(\theta^{1}(t) \| \gamma(t)\right)+\dot{x}_{2}(t) R\left(\theta^{2}(t) \| \gamma(t)\right) .
$$

Here, $\theta_{i, j}^{k}(t)$ is the normalized rate at which balls of color $k$ enter urns that presently contain $i$ balls of color 1 and $j$ balls of color $2, x_{i}(t)$ is the fraction of color- $i$ balls per urn by time $t$, and 
the dot denotes the derivative with respect to time. The overall local rate function also includes an additional term not present in the single-color case, namely the local rate function for the coloring process itself.

In [7], the large deviation upper bound followed from the results in [5], but in the present case this is no longer true since the occupancy process need not be Markov. Instead, we present a direct proof based on weak convergence which only assumes a sample path LDP for the coloration process.

The most significant obstacle to obtaining an LDP occurs in the proof of the large deviation lower bound. The difficulty here is the singular behavior of the relative entropy cost when any element of $\gamma(t)$ approaches zero. In [7], this difficulty was met in two steps. The boundary was avoided everywhere, except at the initial point, using a perturbation argument which relied on the joint convexity of the local rate function. A simple 'filling' construction was then employed in the vicinity of the initial point. The construction is essentially equivalent to the construction of a change of measure with properly bounded Radon-Nikodým derivative that would be needed in the traditional approach to the large deviation lower bound. As mentioned previously, there are simple models for the coloration process (e.g. coloring via a two-state Markov chain) which lead to a local rate function that is not jointly convex and a rate function on path space that is not convex. As a consequence, a more delicate perturbation argument is required, and also the filling construction must be replaced by a more sophisticated argument that is based on time-reversal.

There are a number of applied problems in which occupancy models, and color models in particular, can play an important role. One recent example is in the blocking analysis of circuit-switched networks [6], [10]. Indeed, as shown in [6], the equilibrium distribution of a star network is equivalent to the (conditional) outcome of a randomized occupancy experiment in which a Poisson variable determines the number of balls to be thrown. Star networks are used to model simultaneous service from multiple facilities [12], [16] occurring from different classes of demand as in a telephone network. A demand is allocated a server from each requested facility for a common random duration as long as each facility has a server free at the time of the request. Otherwise, the demand is blocked and cleared. As an occupancy model, the facilities are urns and a ball in an urn corresponds to the allocation of a server to that demand.

In cases when some classes of demand require more than one server from the same facility, a similar equivalence between the equilibrium distribution of the star network and an occupancy experiment can still be established by using a color occupancy model. As an example, one class of demand may require a single server from each of a random pair of facilities and a second class two servers each from a random pair. Color-1 balls are used to indicate demands from the first class at a facility and color-2 balls for demands from the second class [6]. This equivalence allows an LDP to be obtained for the empirical occupancy of the star network (i.e. the fraction of servers with no requests, one request, etc.).

Another recent application for color occupancy problems is the analysis of wavelength conversion in the optical packet switch described in [8]. In each time slot, a random collection of packets (balls) arrive on a set of input fibers and must be routed onto a set of output fibers (urns). The packets on each fiber are wavelength-multiplexed on a finite number of channels (colors). In the absence of wavelength conversion, packets must use the same channel on the input and output fiber. If multiple packets of the same channel belong to the same output fiber, then the excess packets must be converted to a different channel or discarded. Typical quantities to be computed include the probability of requiring a large number of wavelength 
converters and the probability of discarding a large number of packets. The problem was approached with single-color large deviations analysis in [8]; the results there give only an upper bound on the true number of converters because packets discarded due to fiber capacity were also considered to require conversion. By contrast, the multi-color analysis contains the information needed to avoid this overestimation by taking fiber capacity into account. The multicolor approach may also be useful in studying packet switches with constrained wavelength conversion patterns.

Occupancy models play an important role in the analysis of capture/recapture techniques for population estimation, as for example in ecology, pathology, and software fault detection [1], [3], [9], [14], [15]. In this approach, individuals in the population are 'captured' at random and the population size is inferred from the rate at which individuals are 'recaptured'. Assuming that each individual is equally likely to be caught, we obtain an occupancy model with MaxwellBoltzmann statistics, in which the individuals are urns and the number of balls in an urn represents the number of captures. A colored-balls model arises when distinct methods are used to capture individuals. As an illustration, suppose that fish are captured at two locations in a large lake. There may be three distinct subpopulations of fish (those who stay at the east location, those that stay at the west location, and those which swim throughout the lake (the middle fish)). If there are no middle fish, then the two remaining populations may be estimated separately using single-color analysis. If there are only middle fish, then we have no need to distinguish between capture types, and again we may rely on a single color. However, if there are significant numbers of fish of all types, then the analysis of the estimates requires a color model.

The outline of this paper is as follows. In Section 2 a precise formulation of the model is given and the main results of the paper - the upper and lower Laplace principles - are stated. These bounds are equivalent to the large deviation upper and lower bounds. Section 2 also presents three important special cases for coloration processes. In Section 3 the upper bound is established and Section 4 establishes some properties of the rate function which will be needed in the proof of the lower bound in Section 5.

\section{Preliminaries and main result}

We construct an urn model with colored balls as follows. Balls are thrown into one of $n$ urns sequentially. The throwing process is modeled by a collection of i.i.d. random variables

$$
\left\{U_{l}^{n}, l=1, \ldots,\lfloor n \tau\rfloor+1\right\},
$$

where $\lfloor\cdot\rfloor$ denotes the integer-part function. Each $U_{l}^{n}$ is uniformly distributed on the set $\{1, \ldots, n\}$, with each value of the set corresponding to an urn. Thus, a total of $N_{n}:=\lfloor n \tau\rfloor+1$ balls are thrown. There is also a coloration process designated by $Y_{l}^{n} \in\{1,2\}$. At each discrete time a ball is assigned color $Y_{l}^{n}$, and then placed into urn number $U_{l}^{n}$. Thus, $\left\{U_{l}^{n}\right\}$ and $\left\{Y_{l}^{n}\right\}$, respectively, are the random urn and color sequences.

We form empirical measures $\Gamma_{i, j}^{n}(t)$ as follows. If $i \in\{0, \ldots, I\}$ and $j \in\{0, \ldots, J\}$ then

$$
\begin{aligned}
\Gamma_{i, j}^{n}\left(\frac{l}{n}\right):=\frac{1}{n} & \left(\sum_{m=1}^{n} \mathbf{1}\left[\sum_{r=1}^{l} \mathbf{1}\left[U_{r}^{n}=m, Y_{r}^{n}=1\right]=i\right]\right. \\
& \left.\times \mathbf{1}\left[\sum_{r=1}^{l} \mathbf{1}\left[U_{r}^{n}=m, Y_{r}^{n}=2\right]=j\right]\right),
\end{aligned}
$$


where $\mathbf{1}[\cdot]$ is the indicator function. In other words, $\Gamma_{i, j}^{n}(l / n)$ is the fraction of cells containing exactly $i$ color- 1 and $j$ color- 2 balls when $l$ balls have been thrown. Similarly,

$$
\begin{aligned}
& \Gamma_{I+, j}^{n}\left(\frac{l}{n}\right):=\frac{1}{n}( \sum_{m=1}^{n} \mathbf{1}\left[\sum_{r=1}^{l} \mathbf{1}\left[U_{r}^{n}=m, Y_{r}^{n}=1\right]>I\right] \\
&\left.\times \mathbf{1}\left[\sum_{r=1}^{l} \mathbf{1}\left[U_{r}^{n}=m, Y_{r}^{n}=2\right]=j\right]\right), \\
& \Gamma_{i, J+}^{n}\left(\frac{l}{n}\right):=\frac{1}{n}\left(\sum_{m=1}^{n} \mathbf{1}\left[\sum_{r=1}^{l} \mathbf{1}\left[U_{r}^{n}=m, Y_{r}^{n}=1\right]=i\right]\right. \\
&\left.\times \mathbf{1}\left[\sum_{r=1}^{l} \mathbf{1}\left[U_{r}^{n}=m, Y_{r}^{n}=2\right]>J\right]\right), \\
& \Gamma_{I+, J+}^{n}\left(\frac{l}{n}\right):=\frac{1}{n}\left(\sum_{m=1}^{n} \mathbf{1}\left[\sum_{r=1}^{l} \mathbf{1}\left[U_{r}^{n}=m, Y_{r}^{n}=1\right]>I\right]\right. \\
&\left.\times \mathbf{1}\left[\sum_{r=1}^{l} \mathbf{1}\left[U_{r}^{n}=m, Y_{r}^{n}=2\right]>J\right]\right) .
\end{aligned}
$$

By definition,

$$
\Gamma_{0,0}^{n}(0):=1 \quad \text { and } \quad \Gamma_{i, j}^{n}(0):=0
$$

for all other values of $(i, j)$. (We can also consider other initial conditions, with only simple notational changes in the results to be stated below. When extended to accommodate general initial conditions, the large deviation results we will prove are uniform in the initial condition, in the sense used in [7].) The definition of $\Gamma^{n}$ is extended to all $t \in[0, \tau]$ not of the form $l / n$ by piecewise-linear interpolation. Let $\mathcal{M}$ denote the set of all probability measures on $\{0,1, \ldots, I, I+\} \times\{0,1, \ldots, J, J+\}$. The processes $\Gamma^{n}$ are considered to take values in the space of continuous functions $\delta:=C([0, \tau]: \mathcal{M})$, equipped with the usual supremum norm.

We wish to analyze the large deviation asymptotics of these processes, when the underlying coloration process satisfies a large deviations principle and is independent of the urn selection. To this end, it is convenient to use the Laplace formulation. Let $F$ be any bounded and continuous function on $\S$. The processes $\Gamma^{n}$ are said to satisfy a Laplace principle with rate function $I$ if the following two conditions hold:

- for each $M<\infty$, the set $\{\gamma: I(\gamma) \leq M\}$ is compact in $\delta$,

- $\lim _{n \rightarrow \infty}\left[-(1 / n) \log \operatorname{Eexp}\left[-n F\left(\Gamma^{n}\right)\right]\right]=\inf _{\gamma \in s}[I(\gamma)+F(\gamma)]$.

Since the processes $\Gamma^{n}$ take values in a Polish space, the notions of Laplace principle and large deviations principle are equivalent [4, Corollary 1.2.5].

Cumulative coloration processes $\left\{X^{n}, n \in \mathbb{N}\right\}$ are defined for $t=l / n$ by

$$
X_{1}^{n}\left(\frac{l}{n}\right):=\frac{1}{n} \sum_{r=1}^{l} \mathbf{1}\left[Y_{r}^{n}=1\right], \quad X_{2}^{n}\left(\frac{l}{n}\right):=\frac{1}{n} \sum_{r=1}^{l} \mathbf{1}\left[Y_{r}^{n}=2\right] .
$$


These definitions are also extended to $t \in[0, \tau]$ not of the form $l / n$ by piecewise-linear interpolation. Define the set of functions $\mathcal{T}$ by $x=\left(x_{1}, x_{2}\right) \in \mathcal{T}$ if

- $x_{k}(\cdot)$ is increasing and continuous with $x_{k}(0)=0$ for $k=1,2$,

- $x_{1}(t)+x_{2}(t)=t$ for all $t \in[0, \tau]$.

We consider this set of functions as endowed with the usual supremum norm topology, and make the following assumption.

Assumption 1. The sequence of coloration processes $\left\{X^{n}, n \in \mathbb{N}\right\}$ satisfies a large deviations principle on $\mathcal{T}$ with the rate function $J$.

Since $\mathcal{T}$ is also a Polish space, as noted previously, this is equivalent to the statement that $J$ satisfies the following corresponding Laplace principle: $J$ has compact level sets and for all bounded and continuous functions $G: \mathcal{T} \rightarrow \mathbb{R}$ we obtain

$$
\lim _{n \rightarrow \infty}\left[-\frac{1}{n} \log \mathrm{E} \exp \left[-n G\left(X^{n}\right)\right]\right]=\inf _{x \in \mathcal{T}}[J(x)+G(x)] .
$$

Let $\mathcal{C}$ be the set of probability distributions on $\{1,2\}$. In order to prove the large deviation lower bound, we will also need the following stronger assumption.

Assumption 2. Assumption 1 holds and, in addition, the following statements hold.

(a) The rate function $J(x)$ takes the form $\int_{0}^{\tau} M(\dot{x}) \mathrm{d} t$, where $M$ is a proper convex function.

(b) There is a point $a \in \mathcal{C}$ such that $M(a)=0$ and $a_{i}>0, i=1,2$.

The assumed form for $J$ is a special but common case (see the examples below). Since $M$ is a rate function, there is at least one probability vector $a$ at which $M(a)=0$. The assumption that this occurs at a point where both components are positive is very mild.

We next describe a few typical coloration processes. The relative entropy function will be used for this purpose, and indeed throughout this paper. For two probability measures $\alpha$ and $\beta$ on a Polish space $\mathcal{A}$, the relative entropy of $\alpha$ with respect to $\beta$ is defined by

$$
R(\alpha \| \beta):=\int_{\mathcal{A}} \frac{\mathrm{d} \alpha}{\mathrm{d} \beta}\left(\log \frac{\mathrm{d} \alpha}{\mathrm{d} \beta}\right) \mathrm{d} \beta=\int_{\mathcal{A}}\left(\log \frac{\mathrm{d} \alpha}{\mathrm{d} \beta}\right) \mathrm{d} \alpha,
$$

whenever $\alpha$ is absolutely continuous with respect to $\beta$ (and with the convention that $0 \log 0=0$ ). In all other cases, we set $R(\alpha \| \beta)=\infty$.

Example 1. Suppose that we color the balls to achieve a deterministic fraction $p_{k}$ of color $k$, with $p_{k} \in(0,1)$. More precisely, if $N_{l-1}^{k}$ balls of color $k$ have been thrown in the first $l-1$ throws (with $N_{l-1}^{1}+N_{l-1}^{2}=l-1$ ) and if $N_{l-1}^{1} / n \leq p_{1} l / n$, then we color the $l$ th ball 1 , and otherwise color it 2 . The rate function for the corresponding processes $\left\{X^{n}, n \in \mathbb{N}\right\}$ is quite simple:

$$
J(x):= \begin{cases}0 & \text { if } x_{k}(t)=p_{k} t \\ \infty & \text { otherwise. }\end{cases}
$$

Example 2. An alternative coloring scheme is to select the color in an i.i.d. fashion, with probability $p_{k}$ of color $k$, where $p_{k} \in(0,1)$. If $a$ is a probability vector define

$$
M(a):=R(a \| p),
$$


and in all other cases let $M(a):=\infty$. Then the rate function is

$$
J(x):= \begin{cases}\int_{0}^{\tau} M\left(\dot{x}_{1}(t), \dot{x}_{2}(t)\right) \mathrm{d} t & \text { if } x \text { is absolutely continuous } \\ \infty & \text { otherwise. }\end{cases}
$$

Example 3. In our final example the color is determined by a two-state, ergodic Markov process. Let the underlying transition probabilities be denoted by $p_{k, l}, k=1,2, l=1,2$. Let $b$ denote the invariant distribution, with $b_{k} \in(0,1)$. Given a probability vector $a$, let $q_{k, l}$ be any ergodic probability transition matrix with invariant distribution $a$. Define

$$
M(a):=\inf \sum_{k=1}^{2} R\left(q_{k, \cdot} \| p_{k, \cdot}\right) a_{k},
$$

where the infimum is over all such transition matrices $q$. In all other cases set $M(a):=\infty$. Note that $M(a)=0$ if and only if $a=b$. Here again the rate function is written

$$
J(x):= \begin{cases}\int_{0}^{\tau} M\left(\dot{x}_{1}(t), \dot{x}_{2}(t)\right) \mathrm{d} t & \text { if } x \text { is absolutely continuous } \\ \infty & \text { otherwise }\end{cases}
$$

Before turning to the proof of the large deviation result, we introduce the notation needed to define the rate function. Define $\mathcal{V}$ be the set of real $(I+2) \times(J+2)$ matrices, indexed over the set $\{0, \ldots, I+\} \times\{0, \ldots, J+\}$, such that the sum of all elements of each matrix is zero. Let the linear maps $T^{k}: \mathcal{M} \rightarrow \mathcal{V}$ be defined by the expressions

$$
T_{i, j}^{1}[\alpha]=\alpha_{i-1, j}-\alpha_{i, j} \mathbf{1}[i \leq I], \quad T_{i, j}^{2}[\alpha]=\alpha_{i, j-1}-\alpha_{i, j} \mathbf{1}[j \leq J],
$$

where for convenience we define $\alpha_{-1, j}=\alpha_{i,-1}=0$. The map $T^{k}$ is used to determine the rate of change of the urn occupancies, as a function of the occupancy rates $\theta^{k}$ corresponding to balls of color $k$.

Next, let $\gamma \in \delta$ be given with $\gamma_{0,0}(0)=1$. Suppose that there are Borel-measurable functions $\theta^{k}:[0, \tau] \rightarrow \mathcal{M}, k=1,2$, and $x \in \mathcal{T}$ such that, for all $t \in[0, \tau]$,

$$
\gamma(t)=\gamma(0)+\int_{0}^{t}\left(\dot{x}_{1} T^{1}\left[\theta^{1}\right]+\dot{x}_{2} T^{2}\left[\theta^{2}\right]\right) \mathrm{d} s .
$$

Then $I(\gamma)$ is defined by

$$
I(\gamma)=\inf _{x, \theta} \int_{0}^{\tau}\left[\dot{x}_{1} R\left(\theta^{1} \| \gamma\right)+\dot{x}_{2} R\left(\theta^{2} \| \gamma\right)\right] \mathrm{d} s+J(x),
$$

where the infimum is over all such $\theta^{k}$ and $x$ that satisfy (1). If rates satisfying (1) exist with $I(\gamma)<\infty$ then we say that $\gamma$ is a valid occupancy process (or a valid occupancy path). If such rates do not exist, we set $I(\gamma)=\infty$. In Section 3 we show that, for every valid occupancy path, there exist rates $x$ and $\theta^{k}$ which achieve the infimum.

We interpret $\theta_{i, j}^{k}(t)$ as the rate at which balls of color $k$ are thrown into cells that at time $t$ contain $i$ balls of color 1 and $j$ balls of color 2, where the rates are normalized to give a probability measure for each $k$. We follow our usual convention that $i=I+$ refers to more 
than $I$ balls, and likewise for $j=J+$. These normalized rates are modulated by the color selection process $x$ so that $\dot{x}_{k} \theta^{k}$ represents the true rate at which balls of color $k$ enter urns of various occupancy classes. Finally, the transformations $T^{k}[\alpha]$ represent the rate of change in $\gamma$ induced by balls of color $k$ entering urns at the rates given by $\alpha$.

In the next three sections, under different assumptions for the upper and lower bounds, we will prove the Laplace principle for this urn model. In particular, in Section 3 we will prove that

$$
\liminf _{n \rightarrow \infty}\left[-\frac{1}{n} \log \operatorname{E} \exp \left[-n F\left(\Gamma^{n}\right)\right]\right] \geq \inf _{\gamma \in S}[I(\gamma)+F(\gamma)],
$$

and in Section 5 we will prove that

$$
\limsup _{n \rightarrow \infty}\left[-\frac{1}{n} \log \mathrm{E} \exp \left[-n F\left(\Gamma^{n}\right)\right]\right] \leq \inf _{\gamma \in S}[I(\gamma)+F(\gamma)] .
$$

These bounds are equivalent to the large deviation upper and lower bounds, respectively [4, Corollary 1.2.5]. In Section 3 we prove various properties of the rate function $I$, and in particular show that $I$ has compact level sets. Although all statements and proofs are for the case of two colors, there are obvious extensions to the case of any finite number of colors.

To prove these bounds it will be convenient to use a representation for exponential integrals. Let $U^{n}$ and $y^{n}$ denote the product space of $N_{n}:=\lfloor n \tau\rfloor+1$ copies of $\{1, \ldots, n\}$ and $\{1,2\}$, respectively. These are the sets of possible urn sequences and color sequences. Let $\Pi^{n}$ denote the product measure on $U^{n}$, such that each marginal of $\Pi^{n}$ is $\pi^{n}$, the uniform distribution on $\{1, \ldots, n\}$, and let $\Lambda^{n}$ denote the distribution that is induced on $y^{n}$ by the underlying coloring process $\left\{Y_{l}^{n}, l=1, \ldots, N_{n}\right\}$. Let $\mu^{n}$ denote any probability measure on $U^{n} \times y^{n}$. Suppose that $\left\{\bar{U}_{l}^{n}, l=1, \ldots, N_{n}\right\}$ and $\left\{\bar{Y}_{l}^{n}, l=1, \ldots, N_{n}\right\}$ (on the canonical probability space $U^{n} \times y^{n}$ and with expectation operator $\overline{\mathrm{E}}^{n}$ ) have the joint distribution $\mu^{n}$, and that $\bar{\Gamma}^{n}$ and $\bar{X}^{n}$ are constructed from $\left\{\bar{U}_{l}^{n}, l=1, \ldots, N_{n}\right\}$ and $\left\{\bar{Y}_{l}^{n}, l=1, \ldots, N_{n}\right\}$ in exactly the same way that $\Gamma^{n}$ and $X^{n}$ are constructed from $\left\{U_{l}^{n}, l=1, \ldots, N_{n}\right\}$ and $\left\{Y_{l}^{n}, l=1, \ldots, N_{n}\right\}$. Then, from [4, Proposition 1.4.2], we have

$$
-\frac{1}{n} \log \mathrm{E} \exp \left[-n F\left(\Gamma^{n}\right)\right]=\inf _{\mu^{n}} \overline{\mathrm{E}}^{n}\left[\frac{1}{n} R\left(\mu^{n} \| \Pi^{n} \otimes \Lambda^{n}\right)+F\left(\bar{\Gamma}^{n}\right)\right] .
$$

The process $\bar{\Gamma}^{n}$ is an urn model with a 'biased' or 'twisted' distribution. The representation equates the normalized log of the exponential integral with a variational problem, in which we minimize the expected value of the functional $F$ under the twisted distribution, plus a relative entropy 'cost' to achieve the particular twist.

We next present an alternative expression for the relative entropy which reflects the natural relations between the underlying measures. Suppose that $\mu^{n}$ is decomposed into the following product of conditional distributions:

$$
\begin{aligned}
\mu^{n}\left(\mathrm{~d} u_{1}, \ldots, \mathrm{d} u_{N_{n}}, \mathrm{~d} y_{1}, \ldots, \mathrm{d} y_{N_{n}}\right) \\
=\lambda^{n}\left(\mathrm{~d} y_{1}, \ldots, \mathrm{d} y_{N_{n}}\right) \\
\quad \times \mu_{u, 1}^{n}\left(\mathrm{~d} u_{1} \mid y_{1}, \ldots, y_{N_{n}}\right) \cdots \mu_{u, N_{n}}^{n}\left(\mathrm{~d} u_{N_{n}} \mid u_{1}, \ldots, u_{N_{n}-1}, y_{1}, \ldots, y_{N_{n}}\right) .
\end{aligned}
$$

Define the random measures

$$
\bar{\mu}_{l}^{n}\left(\mathrm{~d} u_{l}\right):=\mu_{u, l}^{n}\left(\mathrm{~d} u_{l} \mid \bar{U}_{r}, r=1, \ldots, l-1, \bar{Y}_{r}, r=1, \ldots, N_{n}\right) .
$$


Thus, $\bar{\mu}_{l}^{n}$ is the distribution of the cell into which the $l$ th ball is thrown, given the outcome of all previous throws and the colors of all the balls. Using the fact that $\Pi^{n}$ is a product measure and the chain rule for relative entropy [4, Theorem C.3.1], we have

$$
R\left(\mu^{n} \| \Pi^{n} \otimes \Lambda^{n}\right)=\overline{\mathrm{E}}^{n}\left[\sum_{l=1}^{N_{n}} R\left(\bar{\mu}_{l}^{n} \| \pi^{n}\right)+R\left(\lambda^{n} \| \Lambda^{n}\right)\right] .
$$

This representation separates the total relative entropy into a contribution due to the twisting of the coloration distribution, and a sum of contributions due to twisting of the distribution of the individual throws, conditioned on the coloration process and all previous throws.

\section{The large deviation upper bound}

In this section we prove that

$$
\liminf _{n \rightarrow \infty}\left[-\frac{1}{n} \log \operatorname{E} \exp \left[-n F\left(\Gamma^{n}\right)\right]\right] \geq \inf _{\gamma \in S}[I(\gamma)+F(\gamma)],
$$

which corresponds to the large deviation upper bound. Since in the occupation measure problem we do not distinguish between cells that contain the same number of balls of the various colors, it makes sense to rewrite the relative entropy one last time. Given $\left\{\bar{U}_{r}^{n}, l=1, \ldots, l-1\right\}$ and $\left\{\bar{Y}_{l}^{n}, l=1, \ldots, N_{n}\right\}$, we know that $n \bar{\Gamma}_{i, j}^{n}(l / n)$ is the number of cells that contain $i$ balls of color 1 and $j$ balls of color 2 . For $(i, j), i \in\left\{0,1, \ldots, I^{+}\right\}, j \in\left\{0,1, \ldots, J^{+}\right\}$, let $K_{i, j}$ denote the set of cells of the corresponding type, and let $\left|K_{i, j}\right|=n \bar{\Gamma}_{i, j}^{n}(l / n)$ denote the number of elements of $K_{i, j}$. Let $v_{i, j}^{n}(l / n)$ denote the total probability assigned to cells of this type by $\bar{\mu}_{l}^{n}$ (the definition being irrelevant when $\left|K_{i, j}\right|=0$ ), where

$$
v_{i, j}^{n}\left(\frac{l}{n}\right)=\bar{\mu}_{l}^{n}\left(K_{i, j}\right)=\sum_{m \in K_{i, j}} \bar{\mu}_{l}^{n}(\{m\}) .
$$

The convexity of $x \log x$ then implies the following bound:

$$
\begin{aligned}
\overline{\mathrm{E}}^{n}[R & \left.\left(\bar{\mu}_{l}^{n} \| \pi^{n}\right)\right] \\
& =\overline{\mathrm{E}}^{n}\left[\sum_{i=0, j=0}^{I+, J+} \sum_{m \in K_{i, j}} \log \left(\frac{\bar{\mu}_{l}^{n}(\{m\})}{\pi^{n}(\{m\})}\right) \bar{\mu}_{l}^{n}(\{m\})\right] \\
& \geq \overline{\mathrm{E}}^{n}\left[\sum_{i=0, j=0}^{I+, J+}\left|K_{i, j}\right| \log \left(\frac{\sum_{m \in K_{i, j}} \bar{\mu}_{l}^{n}(\{m\})}{\left|K_{i, j}\right|} \frac{1}{\pi^{n}(\{m\})}\right) \frac{\sum_{m \in K_{i, j}} \bar{\mu}_{l}^{n}(\{m\})}{\left|K_{i, j}\right|}\right] \\
& =\overline{\mathrm{E}}^{n}\left[\sum_{i=0, j=0}^{I+, J+} \log \left(\frac{v_{i, j}^{n}(l / n)}{\bar{\Gamma}_{i, j}^{n}(l / n)}\right) v_{i, j}^{n}\left(\frac{l}{n}\right)\right] \\
& =\overline{\mathrm{E}}^{n}\left[R\left(v^{n}\left(\frac{l}{n}\right) \| \bar{\Gamma}^{n}\left(\frac{l}{n}\right)\right)\right] .
\end{aligned}
$$

The inequality above becomes an equality when the measure $\bar{\mu}_{l}^{n}$ puts the same weight on urns of the same type, and thus we would expect this property to hold for the measure that achieves the minimum in the variational representation. For each $t \in\left[0, N_{n} / n\right]$, define $v^{n}(t)=v^{n}(l / n)$ 
if $t \in[l / n, l / n+1 / n)$. Let $\hat{\Gamma}^{n}$ denote the piecewise-constant (rather than piecewise-linear) interpolant, where

$$
\hat{\Gamma}^{n}(t)=\bar{\Gamma}^{n}\left(\frac{l}{n}\right) \quad \text { for } t \in\left[\frac{l}{n}, \frac{l}{n}+\frac{1}{n}\right) .
$$

Note that if $\bar{\Gamma}^{n}$ converges uniformly to $\bar{\Gamma}$, then so does $\hat{\Gamma}^{n}$.

For a process $\eta$ taking values in $\mathcal{M}$, the set of probabilities on urn types, and for a cumulative coloring $x \in \mathcal{T}$, we define increasing processes $\left(\eta \otimes x_{k}\right)_{i, j}, i \in\{0, \ldots, I, I+\}$, $j \in\{0, \ldots, J, J+\}$, by

$$
\left(\eta \otimes x_{k}\right)_{i, j}(t)=\int_{0}^{t} \eta_{i, j}(s) \dot{x}_{k}(s) \mathrm{d} s .
$$

When $x_{k}(\tau)>0$ and $\left(\eta \otimes x_{k}\right) / x_{k}(\tau)$ appears in the relative entropy function, it is interpreted as the probability measure on $\{0, \ldots, I+\} \times\{0, \ldots, J+\} \times[0, \tau]$ that assigns to the set $A \times B$ the following mass:

$$
\frac{1}{x_{k}(\tau)} \int_{B} \sum_{i, j \in A} \eta_{i, j}(s) \dot{x}_{k}(s) \mathrm{d} s .
$$

Theorem 1. Define the processes $\bar{\Gamma}^{n}, \hat{\Gamma}^{n}, \bar{X}_{k}^{n}, k=1,2$, and $v^{n}$ as above for the given measure $\mu^{n}$. Then the collection

$$
\left\{\left(\bar{\Gamma}^{n}, \bar{X}_{k}^{n}, \hat{\Gamma}^{n} \otimes \bar{X}_{k}^{n}, v^{n} \otimes \bar{X}_{k}^{n}\right), k=1,2, n \in \mathbb{N}\right\}
$$

is tight. Thus, given any subsequence, there exists a further subsequence which converges in distribution to the processes $\bar{\Gamma}, \bar{X}_{k}, \Lambda^{k}, \zeta^{k}, k=1,2$, defined on a probability space with expectation operator $\overline{\mathrm{E}}$. These limit processes have the following properties.

1. Each process $\bar{X}_{k}$ is absolutely continuous (with probability 1 ), with derivative in t denoted by $\dot{\bar{X}}_{k}$.

2. Each process $\zeta^{k}$ can be decomposed in the form

$$
\zeta^{k}=\bar{\theta}^{k} \otimes \bar{X}_{k}
$$

where the measurable process $\bar{\theta}^{k}$ takes values in $\mathcal{M}$.

3. Each process $\Lambda^{k}$ can be decomposed in the form

$$
\Lambda^{k}=\bar{\Gamma} \otimes \bar{X}_{k}
$$

4. The relation (1) holds, with $\gamma, x_{1}, x_{2}, \theta^{1}$, and $\theta^{2}$ replaced by $\bar{\Gamma}, \bar{X}_{1}, \bar{X}_{2}, \bar{\theta}^{1}$, and $\bar{\theta}^{2}$, respectively.

Proof. It is easy to see that the processes $\bar{\Gamma}^{n}, \bar{X}_{k}^{n}, \hat{\Gamma}^{n} \otimes \bar{X}_{k}^{n}$, and $v^{n} \otimes \bar{X}_{k}^{n}, k=1,2$, are all uniformly (in $n$ and $\omega$ ) Lipschitz continuous. Therefore, the ensemble takes values in a compact set, which automatically gives tightness and, hence, convergence along subsequences. If a convergent subsequence is fixed (with limit $\bar{\Gamma}, \bar{X}_{k}, \Lambda^{k}$, and $\zeta^{k}, k=1,2$ ), the limit processes are also Lipschitz continuous and, hence, almost everywhere (in $t$ ) differentiable, with probability 1 . It follows directly from the definitions that $\sum_{i=0, j=0}^{I+, J+} \zeta_{i, j}^{k}(t)=\bar{X}_{k}(t)$ for $t \in[0, \tau]$. Since each component of $\zeta_{i, j}^{k}(t)$ is nondecreasing, there is a measurable, $\mathcal{M}$-valued process $\theta_{i, j}^{k}$ such that

$$
\zeta_{i, j}^{k}(t)=\int_{0}^{t} \theta_{i, j}^{k}(s) \dot{\bar{X}}_{k}(s) \mathrm{d} s .
$$


The convergence of nondecreasing processes $\bar{X}_{k}^{n} \rightarrow \bar{X}_{k}$ and continuity of $\bar{\Gamma}$ imply that $\bar{\Gamma} \otimes \bar{X}_{k}^{n} \rightarrow \bar{\Gamma} \otimes \bar{X}_{k}$. Since $\bar{\Gamma}^{n}$ and, hence, also $\hat{\Gamma}^{n}$ converge uniformly to $\bar{\Gamma}$, we have $\hat{\Gamma}^{n} \otimes \bar{X}_{k}^{n}$ $\rightarrow \bar{\Gamma} \otimes \bar{X}_{k}$. Thus, $\Lambda_{k}$ has the indicated decomposition.

Finally, we consider the last item in the theorem. Consider a component $\bar{\Gamma}_{i, j}$. We assume that $i \in\{1, \ldots, I\}$ and $j \in\{1, \ldots, J\}$, and observe that a similar argument to the one used for this case will give the analogous conclusion for all other cases. Let $\mathcal{F}_{l}^{n}:=\sigma\left(\bar{Y}_{r}^{n}, 1 \leq r \leq N_{n}\right.$, $\left.\bar{U}_{r}^{n}, 1 \leq r \leq l\right)$. We can write

$$
\begin{aligned}
\bar{\Gamma}_{i, j}^{n}\left(\frac{l}{n}+\frac{1}{n}\right)-\bar{\Gamma}_{i, j}^{n}\left(\frac{l}{n}\right) & \\
= & \frac{1}{n} \mathbf{1}\left[\bar{Y}_{k}^{n}=1\right]\left(\mathbf{1}\left[\bar{U}_{k}^{n} \text { is an urn of type }(i-1, j) \text { at time } l\right]\right. \\
& \left.\quad-\mathbf{1}\left[\bar{U}_{k}^{n} \text { is an urn of type }(i, j) \text { at time } l\right]\right) \\
+ & \frac{1}{n} \mathbf{1}\left[\bar{Y}_{k}^{n}=2\right]\left(\mathbf{1}\left[\bar{U}_{k}^{n} \text { is an urn of type }(i, j-1) \text { at time } l\right]\right. \\
= & \frac{1}{n} \mathbf{1}\left[\bar{Y}_{k}^{n}=1\right]\left[v_{i-1, j}^{n}\left(\frac{l}{n}\right)-v_{i, j}^{n}\left(\frac{l}{n}\right)\right] \\
& +\frac{1}{n} \mathbf{1}\left[\bar{Y}_{k}^{n}=2\right]\left[v_{i, j-1}^{n}\left(\frac{l}{n}\right)-v_{i, j}^{n}\left(\frac{l}{n}\right)\right]+e_{i, j}^{n}\left(\frac{l}{n}\right),
\end{aligned}
$$

where $\left\{e_{i, j}^{n}(l / n), l=0, \ldots, N_{n}-1\right\}$ is a martingale difference with respect to $\mathscr{F}_{l}^{n}$ with $\mathrm{E}\left[e_{i, j}^{n}(l / n)\right]^{2}=O\left(1 / n^{2}\right)$. Thus,

$$
\begin{aligned}
\bar{\Gamma}_{i, j}^{n}(t)-\bar{\Gamma}_{i, j}^{n}(0)= & \left(v^{n} \otimes \bar{X}_{1}^{n}\right)_{i-1, j}^{1, n}(t)-\left(v^{n} \otimes \bar{X}_{1}^{n}\right)_{i, j}^{1, n}(t) \\
& +\left(v^{n} \otimes \bar{X}_{2}^{n}\right)_{i, j-1}^{2, n}(t)-\left(v^{n} \otimes \bar{X}_{2}^{n}\right)_{i, j}^{2, n}(t)+g_{i, j}^{n}(t),
\end{aligned}
$$

where the process $g_{i, j}^{n}$ tends uniformly to zero on $[0, \tau]$. Therefore,

$$
\begin{aligned}
\bar{\Gamma}_{i, j}(t)-\bar{\Gamma}_{i, j}(0)= & \left(\theta^{1} \otimes \bar{X}_{1}\right)_{i-1, j}^{1}(t)-\left(\theta^{1} \otimes \bar{X}_{1}\right)_{i, j}^{1}(t) \\
& +\left(\theta^{2} \otimes \bar{X}_{2}\right)_{i, j-1}^{2}(t)-\left(\theta^{2} \otimes \bar{X}_{2}\right)_{i, j}^{2}(t) .
\end{aligned}
$$

The last display is equivalent to the $(i, j)$ th element of (1).

Theorem 2. Under Assumption 1, we have

$$
\liminf _{n \rightarrow \infty}\left[-\frac{1}{n} \log \operatorname{E} \exp \left[-n F\left(\Gamma^{n}\right)\right]\right] \geq \inf _{\gamma \in S}[I(\gamma)+F(\gamma)] .
$$

Proof. Owing to the representation, it suffices to show that

$$
\liminf _{n \rightarrow \infty} \inf _{\mu^{n}} \overline{\mathrm{E}}^{n}\left[\frac{1}{n} R\left(\mu^{n} \| \Pi^{n} \otimes \Lambda^{n}\right)+F\left(\bar{\Gamma}^{n}\right)\right] \geq \inf _{\gamma \in \mathcal{S}}[I(\gamma)+F(\gamma)] .
$$

According to (3) and (5), we have the bound

$$
\frac{1}{n} R\left(\mu^{n} \| \Pi^{n} \otimes \Lambda^{n}\right) \geq \overline{\mathrm{E}}^{n}\left[\frac{1}{n} \sum_{l=1}^{N_{n}} R\left(v^{n}\left(\frac{l}{n}\right) \| \bar{\Gamma}^{n}\left(\frac{l}{n}\right)\right)+\frac{1}{n} R\left(\lambda^{n} \| \Lambda^{n}\right)\right] .
$$


Using the chain rule [4, Theorem C.3.1] again, the nonnegativity of relative entropy, and $\tau \leq N_{n} / n$, we can write

$$
\begin{aligned}
\overline{\mathrm{E}}^{n}\left[\frac{1}{n} \sum_{l=1}^{N_{n}} R\left(v^{n}\left(\frac{l}{n}\right) \| \bar{\Gamma}^{n}\left(\frac{l}{n}\right)\right)\right] \\
\quad \geq \overline{\mathrm{E}}^{n}\left[\int_{0}^{\tau} R\left(v^{n} \| \hat{\Gamma}^{n}\right) \mathrm{d} \bar{X}_{1}^{n}+\int_{0}^{\tau} R\left(v^{n} \| \hat{\Gamma}^{n}\right) \mathrm{d} \bar{X}_{2}^{n}\right] \\
\quad=\overline{\mathrm{E}}^{n}\left[\bar{X}_{1}^{n}(\tau) R\left(\frac{v^{n} \otimes \bar{X}_{1}^{n}}{\bar{X}_{1}^{n}(\tau)} \| \frac{\hat{\Gamma}^{n} \otimes \bar{X}_{1}^{n}}{\bar{X}_{1}^{n}(\tau)}\right)+\bar{X}_{2}^{n}(\tau) R\left(\frac{v^{n} \otimes \bar{X}_{2}^{n}}{\bar{X}_{2}^{n}(\tau)} \| \frac{\hat{\Gamma}^{n} \otimes \bar{X}_{2}^{n}}{\bar{X}_{2}^{n}(\tau)}\right)\right] .
\end{aligned}
$$

According to Theorem 1, given any subsequence of $\mathbb{N}$ we can find a further subsequence (again denoted by $n)$ along which we have the convergence in distribution of $\left(\bar{\Gamma}^{n}, \bar{X}_{k}^{n}, \hat{\Gamma}^{n} \otimes \bar{X}_{k}^{n}\right.$, $\left.v^{n} \otimes \bar{X}_{k}^{n}, k=1,2\right)$. Using Fatou's lemma (for convergence in distribution) and the lower semicontinuity of relative entropy [4, Lemma 1.4.3], we obtain

$$
\begin{aligned}
& \liminf _{n \rightarrow \infty} \overline{\mathrm{E}}^{n}\left[\frac{1}{n} \sum_{l=1}^{N_{n}} R\left(v^{n}\left(\frac{l}{n}\right) \| \bar{\Gamma}^{n}\left(\frac{l}{n}\right)\right)\right] \\
& \quad \geq \liminf _{n \rightarrow \infty} \overline{\mathrm{E}}^{n}\left[\bar{X}_{1}^{n}(\tau) R\left(\frac{v^{n} \otimes \bar{X}_{1}^{n}}{\bar{X}_{1}^{n}(\tau)} \| \frac{\hat{\Gamma}^{n} \otimes \bar{X}_{1}^{n}}{\bar{X}_{1}^{n}(\tau)}\right)+\bar{X}_{2}^{n}(\tau) R\left(\frac{v^{n} \otimes \bar{X}_{2}^{n}}{\bar{X}_{2}^{n}(\tau)} \| \frac{\hat{\Gamma}^{n} \otimes \bar{X}_{2}^{n}}{\bar{X}_{2}^{n}(\tau)}\right)\right] \\
& \quad \geq \overline{\mathrm{E}}\left[\bar{X}_{1}(\tau) R\left(\frac{\bar{\theta}^{1} \otimes \bar{X}_{1}}{\bar{X}_{1}(\tau)} \| \frac{\bar{\Gamma} \otimes \bar{X}_{1}}{\bar{X}_{1}(\tau)}\right)+\bar{X}_{2}(\tau) R\left(\frac{\bar{\theta}^{2} \otimes \bar{X}_{2}}{\bar{X}_{2}(\tau)} \| \frac{\bar{\Gamma} \otimes \bar{X}_{2}}{\bar{X}_{2}(\tau)}\right)\right] \\
& \quad=\overline{\mathrm{E}}\left[\int_{0}^{\tau} R\left(\bar{\theta}^{1}(t) \| \bar{\Gamma}(t)\right) \mathrm{d} \bar{X}_{1}(t)+\int_{0}^{\tau} R\left(\bar{\theta}^{2}(t) \| \bar{\Gamma}(t)\right) \mathrm{d} \bar{X}_{2}(t)\right] .
\end{aligned}
$$

We claim that, since $\left\{X^{n}, n \in \mathbb{N}\right\}$ satisfies a large deviations principle on $\mathcal{T}$ with rate function $J$,

$$
\liminf _{n \rightarrow \infty} \frac{1}{n} R\left(\lambda^{n} \| \Lambda^{n}\right) \geq \overline{\mathrm{E}}[J(\bar{X})] .
$$

Indeed, it follows from the variational representation that, for all bounded and continuous functions $G: \mathcal{T} \rightarrow \mathbb{R}$,

$$
\liminf _{n \rightarrow \infty} \overline{\mathrm{E}}^{n}\left[\frac{1}{n} R\left(\lambda^{n} \| \Lambda^{n}\right)+G\left(\bar{X}^{n}\right)\right] \geq \inf _{x \in \mathcal{T}}[J(x)+G(x)] .
$$

Thus,

$$
\liminf _{n \rightarrow \infty} \frac{1}{n} R\left(\lambda^{n} \| \Lambda^{n}\right) \geq \inf _{x \in \mathcal{T}}[J(x)+G(x)]-\overline{\mathrm{E}}[G(\bar{X})]
$$

and, since $G$ is arbitrary,

$$
\liminf _{n \rightarrow \infty} \frac{1}{n} R\left(\lambda^{n} \| \Lambda^{n}\right) \geq \sup _{G \in \mathcal{C}_{b}(\mathcal{T})}\left[\inf _{x \in \mathcal{T}}[J(x)+G(x)]-\overline{\mathrm{E}}[G(\bar{X})]\right] .
$$

We claim that the right-hand side of this display is bounded below by $\overline{\mathrm{E}}[J(\bar{X})]$. Let $-G_{r}$ be a sequence of bounded, nonnegative, continuous functions that converge up to $J$ as $r \rightarrow \infty$. It follows that $J(x)+G_{r}(x) \geq 0$ for all $r \in[0, \infty)$ and $x \in \mathcal{T}$, and so $\inf _{x \in \mathcal{T}}\left[J(x)+G_{r}(x)\right] \geq 0$. 
Since the monotone convergence theorem implies that $\overline{\mathrm{E}}\left[-G_{r}(\bar{X})\right] \uparrow \overline{\mathrm{E}}[J(\bar{X})]$, the result now follows.

We have the following inequalities, each of which is explained after the display:

$$
\begin{aligned}
& \liminf _{n \rightarrow \infty} {\left[-\frac{1}{n} \log \mathrm{E} \exp \left[-n F\left(\Gamma^{n}\right)\right]\right] } \\
&=\liminf _{n \rightarrow \infty} \overline{\mathrm{E}}^{n}\left[\frac{1}{n} R\left(\mu^{n} \| \Pi^{n} \otimes \Lambda^{n}\right)+F\left(\bar{\Gamma}^{n}\right)\right] \\
& \geq \liminf _{n \rightarrow \infty} \overline{\mathrm{E}}^{n}\left[\frac{1}{n} \sum_{l=1}^{N_{n}} R\left(v^{n}\left(\frac{l}{n}\right) \| \bar{\Gamma}^{n}\left(\frac{l}{n}\right)\right)+\frac{1}{n} R\left(\lambda^{n} \| \Lambda^{n}\right)+F\left(\bar{\Gamma}^{n}\right)\right] \\
& \geq \overline{\mathrm{E}}\left[\int_{0}^{\tau} R\left(\bar{\theta}^{1}(t) \| \bar{\Gamma}(t)\right) \mathrm{d} \bar{X}_{1}(t)+\int_{0}^{\tau} R\left(\bar{\theta}^{2}(t) \| \bar{\Gamma}(t)\right) \mathrm{d} \bar{X}_{2}(t)+J(\bar{X})+F(\bar{\Gamma})\right] \\
& \geq \overline{\mathrm{E}}[I(\bar{\Gamma})+F(\bar{\Gamma})] \\
& \geq \inf _{\gamma \in \delta}[I(\gamma)+F(\gamma)] .
\end{aligned}
$$

Equality (8) is due to the relative entropy representation and the fact that $\mu^{n}$ is a minimizer; inequality (9) uses the decomposition (3) and the bound (5). Inequality (10) uses the bound (7), the bound (6), the convergence in distribution of $\bar{\Gamma}^{n}$ to $\bar{\Gamma}$, and the continuity of $F$. Inequality (11) uses the properties of the limit processes stated in Theorem 1 and the definition of the rate function; inequality (12) is obvious. We have proved that given any subsequence of $\mathbb{N}$ there is a further subsequence along which (4) holds. By the usual argument by contradiction, (4) holds as stated.

\section{Properties of the rate function}

We begin this section by proving some important properties of the rate function; namely compactness of level sets and achievability of the infimizer in the definition of the rate function. The balance of the section develops a series of constructions that are needed in Section 5 to handle the singularity of the rate function at the boundary. In particular, Lemmas 4 and 5, below, show that any path lies close to a path with similar cost which avoids the boundary everywhere except at the initial point. Specifically, each element of the perturbed path is bounded away from zero by a power of $t$. Finally, given such a path, Lemma 6, below, constructs a final perturbed path having piecewise-constant derivatives throughout, and for which the controls are pure (concentrating on one urn type at a time) in a neighborhood of the initial point. This last property is obtained using a reversed-time filling argument. These properties are heavily used in the proof of the large deviation lower bound. The piecewise-constant nature of the controls greatly simplifies the convergence analysis, since we can do the analysis separately on each interval of constancy. In the control representation the occupancy process appears as the second argument in the relative entropy. Since small values would make that cost singular, the lower bound on the trajectories is needed to uniformly control the prelimit costs.

Theorem 3. Under Assumption 1, the set $\{\gamma: I(\gamma) \leq M\}$ is compact for each $M \in[0, \infty)$.

Proof. Since all paths $\gamma$ with $I(\gamma)<\infty$ are Lipschitz continuous with a common constant, we need only show that $\gamma \rightarrow I(\gamma)$ is lower semicontinuous. Let $\gamma_{n} \rightarrow \gamma$ as $n \rightarrow \infty$. If $\liminf _{n \rightarrow \infty} I\left(\gamma_{n}\right)<I(\gamma)$, then we can extract a subsequence (again denoted by $n$ ) such that 
$I\left(\gamma_{n}\right)$ converges and $\lim _{n \rightarrow \infty} I\left(\gamma_{n}\right)=I(\gamma)-\varepsilon$, for some $\varepsilon>0$. Let $\theta^{k, n}, k=1,2$, and $x^{n}$ be associated rates and cumulative coloration processes that satisfy

$$
\dot{\gamma}_{n}=\dot{x}_{1}^{n} T^{1}\left[\theta^{1, n}\right]+\dot{x}_{2}^{n} T^{2}\left[\theta^{2, n}\right]
$$

and

$$
I\left(\gamma_{n}\right)=\int_{0}^{\tau}\left[\dot{x}_{1}^{n} R\left(\theta^{1, n} \| \gamma_{n}\right)+\dot{x}_{2}^{n} R\left(\theta^{2, n} \| \gamma_{n}\right)\right] \mathrm{d} t+J\left(x^{n}\right)+O\left(\frac{1}{n}\right) .
$$

Exactly as in the proof of the convergence theorem (Theorem 1), the uniformly Lipschitz continuous processes $\left(\gamma_{n}, x_{k}^{n}, \theta^{k, n} \otimes x_{k}^{n}, \gamma_{n} \otimes x_{k}^{n}, k=1,2\right)$ converge, at least along a subsequence, to a collection of processes $\left(\gamma, x_{k}, \theta^{k} \otimes x_{k}, \gamma \otimes x_{k}, k=1,2\right)$. Using the lower semicontinuity of $J$ and the relative entropy, we obtain

$$
\begin{aligned}
\liminf _{n \rightarrow \infty}\left[\int_{0}^{\tau}\left[\dot{x}_{1}^{n} R\left(\theta^{1, n} \| \gamma_{n}\right)+\dot{x}_{2}^{n} R\left(\theta^{2, n} \| \gamma_{n}\right)\right] \mathrm{d} t+J\left(x^{n}\right)\right] \\
=\liminf _{n \rightarrow \infty}\left[\int _ { 0 } ^ { \tau } \left[x_{1}^{n}(\tau) R\left(\frac{\theta^{1, n} \otimes x_{1}^{n}}{x_{1}^{n}(\tau)} \| \frac{\gamma_{n} \otimes x_{1}^{n}}{x_{1}^{n}(\tau)}\right)\right.\right. \\
\left.\left.\quad+x_{2}^{n}(\tau) R\left(\frac{\theta^{2, n} \otimes x_{2}^{n}}{x_{2}^{n}(\tau)} \| \frac{\gamma_{n} \otimes x_{2}^{n}}{x_{2}^{n}(\tau)}\right)\right] \mathrm{d} t+J\left(x^{n}\right)\right] \\
\geq \int_{0}^{\tau}\left[x_{1}(\tau) R\left(\frac{\theta^{1} \otimes x_{1}}{x_{1}(\tau)} \| \frac{\gamma \otimes x_{1}}{x_{1}(\tau)}\right)+x_{2}(\tau) R\left(\frac{\theta^{2} \otimes x_{2}}{x_{2}(\tau)} \| \frac{\gamma \otimes x_{2}}{x_{2}(\tau)}\right)\right] \mathrm{d} t+J(x) \\
=\int_{0}^{\tau}\left[\dot{x}_{1} R\left(\theta^{1} \| \gamma\right)+\dot{x}_{2} R\left(\theta^{2} \| \gamma\right)\right] \mathrm{d} t+J(x) .
\end{aligned}
$$

Since we also have

$$
\begin{aligned}
\gamma(t)-\gamma(0) & =\lim _{n \rightarrow \infty}\left[\gamma_{n}(t)-\gamma_{n}(0)\right] \\
& =\lim _{n \rightarrow \infty} \int_{0}^{t}\left[\dot{x}_{1}^{n} T^{1}\left[\theta^{1, n}\right]+\dot{x}_{2}^{n} T^{2}\left[\theta^{2, n}\right]\right] \mathrm{d} s \\
& =\lim _{n \rightarrow \infty}\left[T^{1}\left[\int_{0}^{t} \dot{x}_{1}^{n} \theta^{1, n} \mathrm{~d} s\right]+T^{2}\left[\int_{0}^{t} \dot{x}_{2}^{n} \theta^{2, n} \mathrm{~d} s\right]\right] \\
& =T^{1}\left[\int_{0}^{t} \dot{x}_{1} \theta^{1} \mathrm{~d} s\right]+T^{2}\left[\int_{0}^{t} \dot{x}_{2} \theta^{2} \mathrm{~d} s\right] \\
& =\int_{0}^{t}\left[\dot{x}_{1} T^{1}\left[\theta^{1}\right]+\dot{x}_{2} T^{2}\left[\theta^{2}\right]\right] \mathrm{d} s
\end{aligned}
$$

we conclude that

$$
I(\gamma) \leq \int_{0}^{\tau}\left[\dot{x}_{1} R\left(\theta^{1} \| \gamma\right)+\dot{x}_{2} R\left(\theta^{2} \| \gamma\right)\right] \mathrm{d} t+J(x) \leq \lim _{n \rightarrow \infty} I\left(\gamma_{n}\right),
$$

which is a contradiction. Therefore, $\liminf _{n \rightarrow \infty} I\left(\gamma_{n}\right) \geq I(\gamma)$.

It is also true that, given any $\gamma$, infimizing $\theta$ s and $x$ s exist.

Lemma 1. Let $\gamma \in \&$ be given. There exist measurable functions $\theta^{k}, k=1,2$, and $x \in \mathcal{T}$ which achieve the infimum in the definition of $I(\gamma)$. 
Proof. Since the proof uses the same ideas as that of Theorem 3, the argument is only sketched. If $I(\gamma)=\infty$ then there is nothing to prove. If $I(\gamma)<\infty$ then there exist $\theta^{k, n}$, $k=1,2$, and $x^{n} \in \mathcal{T}$ such that

$$
\dot{\gamma}=\dot{x}_{1}^{n} T^{1}\left[\theta^{1, n}\right]+\dot{x}_{2}^{n} T^{2}\left[\theta^{2, n}\right]
$$

and

$$
J^{n}(x)+\int_{0}^{\tau}\left[\dot{x}_{1}^{n} R\left(\theta^{1, n} \| \gamma\right)+\dot{x}_{2}^{n} R\left(\theta^{2, n} \| \gamma\right)\right] \mathrm{d} t \leq I(\gamma)+O\left(\frac{1}{n}\right) .
$$

Arguing exactly as in Theorem 3, we can consider the limit as $n \rightarrow \infty$ (along a subsequence) and construct the minimizing $\theta^{k}, k=1,2$, and $x \in \mathcal{T}$.

Recall that Assumption 2 specifies the common case when the coloration rate function takes the form

$$
J(x)=\int_{0}^{\tau} M\left(\dot{x}_{1}, \dot{x}_{2}\right) \mathrm{d} t
$$

for some convex proper function $M: \mathbb{R}^{2} \rightarrow[0, \infty]$. In this case we can write $I$ as

$$
I(\gamma)=\int_{0}^{\tau} L(\gamma, \dot{\gamma}) \mathrm{d} t
$$

with the local rate function

$$
\begin{aligned}
L(\gamma, \eta):=\inf \left\{a_{1} R\left(\theta^{1} \| \gamma\right)+a_{2} R\left(\theta^{2} \| \gamma\right)+M\left(a_{1}, a_{2}\right):\right. & \\
a & \left.\in \mathcal{C}, \theta^{k} \in \mathcal{M}, \eta=a_{1} T^{1}\left[\theta^{1}\right]+a_{2} T^{2}\left[\theta^{2}\right]\right\},
\end{aligned}
$$

where $\mathcal{C}$ is the set of probability distributions on $\{1,2\}$.

In the remainder of this section we will construct processes and controls that will be used in the proof of the large deviation lower bound. We first define the natural occupancy path corresponding to a given colorization process, and the zero-cost path. For $y \in[0, \infty)$ and $i \in \mathbb{Z}$, let

$$
\mathcal{P}_{i}(y)= \begin{cases}\frac{y^{i}}{i !} \mathrm{e}^{-y}, & i \geq 0, \\ 0, & i<0,\end{cases}
$$

denote the $i$ th component of a Poisson distribution with mean $y$, and let $\mathcal{Q}_{i}(y)=\sum_{j>i} \mathcal{P}_{j}(y)$ be the Poisson tail probability function. The product of two independent Poisson distributions with means $y_{1}$ and $y_{2}$ defines a mapping $\Phi: \mathbb{R}^{+} \times \mathbb{R}^{+} \rightarrow \mathcal{M}$ given by

$$
\begin{aligned}
\Phi_{i, j}\left(y_{1}, y_{2}\right) & =\mathcal{P}_{i}\left(y_{1}\right) \mathcal{P}_{j}\left(y_{2}\right), \\
\Phi_{i, J+}\left(y_{1}, y_{2}\right) & =\mathcal{P}_{i}\left(y_{1}\right) \mathcal{Q}_{J}\left(y_{2}\right), \\
\Phi_{I+, j}\left(y_{1}, y_{2}\right) & =\mathcal{Q}_{I}\left(y_{1}\right) \mathcal{P}_{j}\left(y_{2}\right), \\
\Phi_{I+, J+}\left(y_{1}, y_{2}\right) & =\mathcal{Q}_{I}\left(y_{1}\right) \mathcal{Q}_{J}\left(y_{2}\right) .
\end{aligned}
$$

The mapping $\Phi$ is the limiting (as $n \rightarrow \infty$ ) mean urn occupancy distribution for an experiment in which $n y_{k}$ balls of color $k$ are thrown into $n$ urns.

Lemma 2. (Natural occupancy path.) Suppose that Assumption 2(a) holds. Let $x \in \mathcal{T}$ be a colorization process with finite cost $J(x)$. The natural occupancy path corresponding to $x$ defined by $\gamma^{*}(t)=\Phi\left(x_{1}(t), x_{2}(t)\right)$ is an occupancy path in $\&$ which satisfies the initial condition $\gamma_{0,0}^{*}(0)=1$ and the bound $I\left(\gamma^{*}\right) \leq J(x)$. 
Proof. The initial condition is immediate from the fact that $x(0)=0$. The continuity of $x$ and of the Poisson distribution with respect to its mean ensure that $\gamma^{*} \in \S$. To establish the bound on $I\left(\gamma^{*}\right)$, we will show that the derivative of the path satisfies the differential equation

$$
\dot{\gamma}^{*}=\dot{x}_{1} T^{1}\left[\gamma^{*}\right]+\dot{x}_{2} T^{2}\left[\gamma^{*}\right] .
$$

Then, according to the definition of the rate function, we obtain

$$
I\left(\gamma^{*}\right) \leq \int_{0}^{\tau}\left(\sum_{k=1}^{2} \dot{x}_{k} R\left(\gamma^{*} \| \gamma^{*}\right)+M(\dot{x})\right) \mathrm{d} t=\int_{0}^{\tau} M(\dot{x}) \mathrm{d} t=J(x) .
$$

Note that $(\mathrm{d} / \mathrm{d} x) \mathcal{P}_{i}(x)=\mathcal{P}_{i-1}(x)-\mathcal{P}_{i}(x)$. Then, for $i \leq I$ and $j \leq J$, we have

$$
\begin{aligned}
\dot{\gamma}_{i, j}^{*} & =\dot{x}_{1}\left[\mathcal{P}_{i-1}\left(x_{1}\right)-\mathcal{P}_{i}\left(x_{1}\right)\right] \mathcal{P}_{j}\left(x_{2}\right)+\dot{x}_{2} \mathcal{P}_{i}\left(x_{1}\right)\left[\mathcal{P}_{j-1}\left(x_{2}\right)-\mathcal{P}_{j}\left(x_{2}\right)\right] \\
& =\dot{x}_{1}\left(\gamma_{i-1, j}^{*}-\gamma_{i, j}^{*}\right)+\dot{x}_{2}\left(\gamma_{i, j-1}^{*}-\gamma_{i, j}^{*}\right),
\end{aligned}
$$

as required. Using the fact that $(\mathrm{d} / \mathrm{d} x) \mathcal{Q}_{i}(x)=\mathscr{P}_{i}(x)$, the cases involving $i=I+$ or $j=J+$ follow in a similar manner.

The following lemma is immediate, using the linear colorization process $x(t)=a t$.

Lemma 3. (Zero-cost path.) Suppose that Assumption 2 holds, with $a \in \mathcal{C}$ such that $M(a)=0$. Then the function $z(t)=\Phi\left(a_{1} t, a_{2} t\right)$ is an occupancy path in $\&$ which satisfies $I(z)=0$ and the initial condition $z_{0,0}(0)=1$.

In the single-color case analyzed in [7], the local rate function expressed by (13) takes the simpler form

$$
L_{\mathrm{s}}(\gamma, \eta)=R(\theta \| \gamma)
$$

where $\gamma$ and $\theta$ are probability distributions on $\{0, \ldots, I+\}$ and where $\theta$ has the explicit form $\theta_{i}=-\sum_{j=0}^{i} \eta_{j}$. In that case, the convexity of the relative entropy implies that the local rate function is a convex function of its arguments.

In the present case, the local rate function is a convex function of $\eta$ but is not necessarily jointly convex in $(\gamma, \eta)$. It can in fact be shown that, under Assumption $2, L$ is convex if and only if the function $M(a)+h(a)$ is convex for $a \in \mathcal{C}$, where $h(a)=-a_{1} \log a_{1}-a_{2} \log a_{2}$ is the entropy function. It is easy to see that Examples 1 and 2 always satisfy this condition. However, it can also be demonstrated that Example 3 satisfies this condition if and only if the Markov transition probabilities satisfy $p_{11}+p_{22} \leq 1$.

In our proof of the lower bound, we require a technical result showing that every valid occupancy path $\gamma$ is close, both in supremum norm and in cost, to an occupancy path for which each element is bounded away from zero by a power of $t$. This fact allows us to avoid explictly considering occupancy paths which are close to the boundary after time $t=0$. When the local rate function is convex, this technical result is easily demonstrated by slightly perturbing the given occupancy path in the direction of the zero-cost path (which itself avoids the boundary after $t=0$ ).

We will use a modified form of this argument to establish this result without requiring convexity of the local rate function. We first show that $\gamma$ is close to an occupancy path $\hat{\gamma}$ whose optimal colorization process $\hat{x}$ has each component $\hat{x}_{k}(t)$ bounded below by a function of the form $\delta t$ for some $\delta>0$. Secondly, we show that perturbing $\hat{\gamma}$ in the direction of the natural occupancy process $\gamma^{*}$ corresponding to $\hat{x}$ yields an occupancy process with the desired 
properties, namely being close to $\gamma$ in supremum norm and cost and having each urn fraction bounded away from zero by a power of time $t$. These steps are undertaken in the following two lemmas. Let $d$ denote the supremum norm distance.

Lemma 4. Suppose that Assumption 2 holds. Let $\gamma \in \delta$ be given and let $\varepsilon>0$. There exists $\hat{\gamma} \in \delta$, with $I(\hat{\gamma})<\infty$ and associated optimal rates $\hat{x}$ and $\hat{\theta}$, which satisfies the properties

- $I(\hat{\gamma}) \leq I(\gamma)+\varepsilon$,

- $d(\gamma, \hat{\gamma}) \leq \varepsilon$,

- there exists $\delta>0$ such that $\hat{x}_{k}(t) \geq \delta$ for all $t \in[0, \tau]$.

Proof. We will construct $\hat{\gamma}$ by following the zero-cost path $z$ of Lemma 3 for a short time $\Delta$ and then closely tracking the original process $\gamma$ for $t>\Delta$. The key step is to construct an occupancy process $\tilde{\gamma}$ which has initial condition $\tilde{\gamma}(0)=z(\Delta)$ and which for sufficiently small $\Delta$ satisfies $d(\gamma, \tilde{\gamma}) \leq \varepsilon / 2$ and $I(\tilde{\gamma}) \leq I(\gamma)+\varepsilon$. Once $\tilde{\gamma}$ has been constructed, we may then define

$$
\hat{\gamma}(t)= \begin{cases}z(t), & 0 \leq t \leq \Delta, \\ \tilde{\gamma}(t-\Delta), & \Delta<t \leq \tau .\end{cases}
$$

Since the absolute value of the derivative of each element of an occupancy function with finite cost is bounded by 1 , we immediately have $d(\hat{\gamma}, \tilde{\gamma}) \leq \Delta$ and, hence, $d(\hat{\gamma}, \gamma)<\varepsilon$ for sufficiently small $\Delta$. Moreover,

$$
I(\hat{\gamma})=0+\int_{0}^{\tau-\Delta} L(\tilde{\gamma}, \dot{\tilde{\gamma}}) \mathrm{d} t \leq I(\tilde{\gamma}) \leq I(\gamma)+\varepsilon .
$$

Finally, the optimal colorization rate for $\hat{\gamma}$ on the interval $[0, \Delta]$ is given by $\hat{x}_{k}(t)=a_{k} t$. Because the colorization processes are monotonically increasing, we have the bound $\hat{x}_{k}(t) \geq$ $\left(a_{k}(\Delta \wedge \tau) / \tau\right) t$ for all $t \in[0, \tau]$.

It remains to construct an occupancy function $\tilde{\gamma}$ with the required properties. We define

$$
\tilde{\gamma}(t)=\mathrm{e}^{-\Delta}(\gamma(t)-\gamma(0))+z(\Delta)=\mathrm{e}^{-\Delta} \gamma(t)+\left(1-\mathrm{e}^{-\Delta}\right) z_{\mathrm{c}}(\Delta),
$$

where $z(\Delta)$ is the zero-cost distribution of Lemma 3 at time $\Delta$ and

$$
z_{\mathrm{c}}(\Delta)=\frac{z(\Delta)-\mathrm{e}^{-\Delta} \gamma(0)}{1-\mathrm{e}^{-\Delta}}
$$

is the conditional distribution obtained from $z(\Delta)$ by removing the probability mass from its $(0,0)$ th element. As $\tilde{\gamma}$ is a convex combination of two elements of $\delta$, we immediately have $\tilde{\gamma} \in \delta$, and it is also clear that $d(\tilde{\gamma}, \gamma)$ can be made arbitrarily small by decreasing $\Delta$.

It remains to establish the desired bound on $I(\tilde{\gamma})$. If $\alpha \in \mathcal{M}$ is the distribution that puts all of its mass on the $(I+, J+)$ th element, note that $T^{k}[\alpha]=0$ for $k=1,2$, reflecting that balls thrown into $I+$ or $J+$ urns have no effect on the occupancy state. Let $x$ and $\theta$ be the optimal rate processes for $\gamma$. Then the rates $\tilde{x}=x$ and $\tilde{\theta}^{k}=\mathrm{e}^{-\Delta} \theta^{k}+\left(1-\mathrm{e}^{-\Delta}\right) \alpha$ satisfy

$$
\dot{\tilde{x}}_{1} T^{1}\left[\tilde{\theta}^{1}\right]+\dot{\tilde{x}}_{2} T^{2}\left[\tilde{\theta}^{2}\right]=\mathrm{e}^{-\Delta}\left(\dot{x}_{1} T^{1}\left[\theta^{1}\right]+\dot{x}_{2} T^{2}\left[\theta^{2}\right]\right)=\mathrm{e}^{-\Delta} \dot{\gamma}=\dot{\tilde{\gamma}},
$$


and are therefore feasible rates for constructing $\tilde{\gamma}$. Using $\tilde{x}=x$ and the joint convexity of the relative entropy, we have

$$
\begin{aligned}
I(\tilde{\gamma}) \leq & \int_{0}^{\tau} \sum_{k=1}^{2} \dot{\tilde{x}}_{k} R\left(\tilde{\theta}^{k} \| \tilde{\gamma}\right) \mathrm{d} t+J(\tilde{x}) \\
\leq & \mathrm{e}^{-\Delta} \int_{0}^{\tau} \sum_{k=1}^{2} \dot{x}_{k} R\left(\theta^{k} \| \gamma\right) \mathrm{d} t \\
& +\left(1-\mathrm{e}^{-\Delta}\right) \int_{0}^{\tau} \sum_{k=1}^{2} \dot{x}_{k} R\left(\alpha \| z_{\mathrm{c}}(\Delta)\right) \mathrm{d} t+J(x) \\
\leq & I(\gamma)+\tau\left(1-\mathrm{e}^{-\Delta}\right) R\left(\alpha \| z_{\mathrm{c}}(\Delta)\right) .
\end{aligned}
$$

Using the bound $z_{I+, J+}(\Delta) \geq \mathcal{P}_{I+1}\left(a_{1} \Delta\right) \mathcal{P}_{J+1}\left(a_{2} \Delta\right)$, the difference $I(\tilde{\gamma})-I(\gamma)$ is bounded by

$$
\tau\left(1-\mathrm{e}^{-\Delta}\right) \log \left(a_{1}^{-I-1} a_{2}^{-J-1} \Delta^{-(I+J+2)}(I+1) !(J+1) ! \mathrm{e}^{\Delta}\left(1-\mathrm{e}^{-\Delta}\right)\right),
$$

which approaches zero as $\Delta \rightarrow 0$.

Lemma 5. Suppose that Assumption 2 holds. Let $\gamma \in \&$ be given such that $I(\gamma)<\infty$, and let $\varepsilon>0$. Then there exist $\delta>0, K \in \mathbb{N}$, and $\gamma^{\varepsilon} \in \delta$ with the properties

(a) $I\left(\gamma^{\varepsilon}\right) \leq I(\gamma)+\varepsilon$,

(b) $d\left(\gamma^{\varepsilon}, \gamma\right) \leq \varepsilon$,

(c) $\gamma_{i, j}^{\varepsilon}(t)>\delta t^{K}$ for all $t \in(0, \tau]$ and $(i, j) \in\{0,1, \ldots, I, I+\} \times\{0,1, \ldots, J, J+\}$.

Proof. Using Lemma 4, there exist $\hat{\gamma}$ and $\bar{\delta}>0$ with $d(\gamma, \hat{\gamma})<\varepsilon / 2, I(\hat{\gamma}) \leq I(\gamma)+\varepsilon$, and $\hat{x}_{k} \geq \bar{\delta} t$, where $\hat{x}$ and $\hat{\theta}$ are the optimal rates used in the definition of $I(\hat{\gamma})$. Let $\gamma^{*}=\Phi\left(\hat{x}_{1}, \hat{x}_{2}\right)$ be the natural occupancy path corresponding to $\hat{x}$, and define

$$
\gamma^{\varepsilon}=(1-\lambda) \hat{\gamma}+\lambda \gamma^{*}
$$

By the triangle inequality, $\gamma^{\varepsilon}$ will be sufficiently close to $\gamma$ if $\lambda<\varepsilon / 4$. Note that the rates $x^{\varepsilon}=\hat{x}$ and $\theta^{k, \varepsilon}=(1-\lambda) \hat{\theta}^{k}+\lambda \gamma^{*}$ are feasible rates for generating $\dot{\gamma}^{\varepsilon}$, so that

$$
\begin{aligned}
I\left(\gamma^{\varepsilon}\right) & \leq \int_{0}^{\tau} \sum_{k=1}^{2} \dot{\hat{x}}_{k} R\left(\theta^{k, \varepsilon} \| \gamma^{\varepsilon}\right) \mathrm{d} t+J(\hat{x}) \\
& \leq(1-\lambda) I(\hat{\gamma})+\lambda I\left(\gamma^{*}\right) \\
& \leq I(\hat{\gamma}) \\
& \leq I(\gamma)+\varepsilon
\end{aligned}
$$

where we have used convexity of the relative entropy and the fact that $I\left(\gamma^{*}\right)=J(\hat{x}) \leq I(\hat{\gamma})$. 
Finally, for $i \leq I$ and $j \leq J$, the $(i, j)$ th element of $\gamma^{\varepsilon}$ satisfies the lower bound

$$
\begin{aligned}
\gamma_{i, j}^{\varepsilon}(t) & \geq \lambda \gamma_{i, j}^{*}(t) \\
& =\lambda \frac{\hat{x}_{1}^{i}(t) \hat{x}_{2}^{j}(t)}{i ! j !} \mathrm{e}^{-t} \\
& \geq \lambda \frac{\bar{\delta}^{i+j} \mathrm{e}^{-\tau}}{i ! j !} t^{i+j},
\end{aligned}
$$

for all $t \in[0, \tau]$. Similar bounds hold for cases involving $i=I+$ and $j=J+$, since $\mathcal{Q}_{i} \geq \mathcal{P}_{i+1}$. Hence, $\gamma^{\varepsilon}$ has all of the desired properties.

The final lemma of this section is the principle result that will be used to support the proof of the lower bound.

Lemma 6. Suppose that Assumption 2 holds. Let $\gamma \in \&$ be given such that $I(\gamma)<\infty$ and such that for some $\delta>0$ and $K \in \mathbb{N}$ the lower bound $\gamma_{i, j}(t) \geq \delta t^{K}$ holds for all $(i, j) \in\{0,1, \ldots, I, I+\} \times\{0,1, \ldots, J, J+\}$. Let $x, \theta^{1}$, and $\theta^{2}$ satisfy

$$
\dot{\gamma}=\dot{x}_{1} T^{1}\left[\theta^{1}\right]+\dot{x}_{2} T^{2}\left[\theta^{2}\right]
$$

and

$$
I(\gamma)=\int_{0}^{\tau}\left[\dot{x}_{1} R\left(\theta^{1} \| \gamma\right)+\dot{x}_{2} R\left(\theta^{2} \| \gamma\right)\right] \mathrm{d} t+J(x) .
$$

Given $\varepsilon>0$ there exist $\gamma^{*}, \theta^{1, *}, \theta^{2, *}$, and $\sigma>0$ with the following properties.

1. $\dot{\gamma}^{*}=\dot{x}_{1} T^{1}\left[\theta^{1, *}\right]+\dot{x}_{2} T^{2}\left[\theta^{2, *}\right]$ and $\gamma_{0,0}^{*}(0)=1$.

2. $I\left(\gamma^{*}\right) \leq \int_{0}^{\tau}\left[\dot{x}_{1} R\left(\theta^{1, *} \| \gamma_{i, j}^{*}\right)+\dot{x}_{2} R\left(\theta^{2, *} \| \gamma_{i, j}^{*}\right)\right] \mathrm{d} t+J(x) \leq I(\gamma)+\varepsilon$.

3. $d\left(\gamma^{*}, \gamma\right) \leq \varepsilon$.

4. The rate processes $\theta^{1, *}$ and $\theta^{2, *}$ are piecewise constant on $[0, \tau]$, with a finite number of intervals of constancy.

5. When restricted to $[0, \sigma)$, the rate processes are pure in the sense that on any interval of constancy $\left(s_{1}, s_{2}\right)$, and for each $k=1,2$, there exists $(i, j) \in\{0,1, \ldots, I, I+\} \times$ $\{0,1, \ldots, J, J+\}$ such that $\theta_{i, j}^{k, *}(t)=1$ for all $t \in\left(s_{1}, s_{2}\right)$. In addition, for $k=1,2$ and any interval of constancy on which $\theta_{i, j}^{k, *}(t)=1, \gamma_{i, j}^{*}(t) \geq \delta \sigma^{K}$.

Proof. Suppose that $\gamma, \theta^{1}, \theta^{2}$, and $x$ satisfy the assumptions of the lemma. Let $\sigma \in(0, \tau]$. By assumption, we have $\gamma_{i, j}(\sigma) \geq \delta \sigma^{K}$. We can choose $\sigma>0$ such that $-\sigma \log \left(\delta \sigma^{K}\right) \leq \varepsilon / 2$, and such that if $\gamma_{1}$ and $\gamma_{2}$ are any occupancy processes with the same initial condition, then $\left\|\gamma_{1}(s)-\gamma_{2}(s)\right\| \leq \varepsilon$ for all $s \in[0, \sigma]$ (here we use the common Lipschitz continuity for all occupancy functions). A time-reversed induction argument will be used to construct the pure controls on $[0, \sigma)$ described in the lemma. The main idea is that the occupancy path $\gamma^{*}$ will proceed on $[0, \sigma]$ in such a way that each element increases to a maximum level before decreasing to its final value, $\gamma^{*}(\sigma)=\gamma(\sigma)$. Hence, the contents of any given occupancy class are only reduced at times when that class has at least a fraction $\delta \sigma^{K}$ of the urns. In other words, $\theta_{i, j}^{k, *}(s)>0$ implies that $\gamma_{i, j}^{*}(s) \geq \delta \sigma^{K}$, allowing the cost of $\gamma^{*}$ on $(0, \sigma)$ to be made arbitrarily small. 
For a given $\gamma \in \mathcal{M}$, the associated minimum number of balls of each color per urn is given by applying the linear operators

$$
\begin{aligned}
& \beta_{1}^{\mathrm{e}}(\gamma)=\sum_{i=0}^{I} \sum_{j=0}^{J+} i \gamma_{i, j}+\sum_{j=0}^{J+}(I+1) \gamma_{I+, j}, \\
& \beta_{2}^{\mathrm{e}}(\gamma)=\sum_{j=0}^{J} \sum_{i=0}^{I+} j \gamma_{i, j}+\sum_{i=0}^{I+}(J+1) \gamma_{i, J+}
\end{aligned}
$$

(these are only the minimum number per urn because balls may fall into urns with more than $I+1$ or $J+1$ of color 1 or color 2 , respectively). Because $x$ is a coloring process which generates $\gamma(\sigma)$, it must satisfy $x_{k}(\sigma) \geq \beta_{k}^{\mathrm{e}}(\gamma(\sigma))$. As another way to see this point, we may verify the relations

$$
\begin{array}{ll}
\beta_{1}^{\mathrm{e}}\left(T^{1}[\theta]\right)=\sum_{i=0}^{I} \sum_{j=0}^{J+} \theta_{i, j}, & \beta_{1}^{\mathrm{e}}\left(T^{2}[\theta]\right)=0, \\
\beta_{2}^{\mathrm{e}}\left(T^{1}[\theta]\right)=0, & \beta_{2}^{\mathrm{e}}\left(T^{2}[\theta]\right)=\sum_{i=0}^{I+} \sum_{j=0}^{J} \theta_{i, j} .
\end{array}
$$

The definition of $\dot{\gamma}$ in terms of $\dot{x}$ and $\theta$ then implies that $0 \leq \dot{\beta}_{k}^{\mathrm{e}}(\gamma) \leq \dot{x}_{k}$.

To simplify the exposition, we first assume that $x_{k}(\sigma)=\beta_{k}^{\mathrm{e}}(\gamma(\sigma))$, and subsequently extend the argument to cover inequality.

For colors $k=1,2$, we define orderings $\xi_{k}:\{0, \ldots, I+\} \times\{0, \ldots, J+\} \rightarrow N$ by

$$
\xi_{1}(i, j)=(J+1) i+j, \quad \xi_{2}(i, j)=i+(I+1) j,
$$

where strictly speaking we substitute $i=I+1$ or $j=J+1$ on the right-hand side when $i=I+$ or $j=J+$ appears on the left-hand side. Observe that each class of urns corresponds to a distinct value of $\xi_{k}$, and that each of these functions induces a strict ordering on $\{0, \ldots, I, I+\} \times$ $\{0, \ldots, J, J+\}$. For any $\gamma \in \mathcal{M}$, let $U(\gamma)$ be the set of pairs $(i, j)$ such that $\gamma_{i, j}>0$, and for $k=1,2$ define

$$
\begin{aligned}
\kappa_{k}(\gamma) & =\max _{U(\gamma)} \xi_{k}(i, j), \\
u_{k}(\gamma) & =\underset{U(\gamma)}{\arg \max } \xi_{k}(i, j) .
\end{aligned}
$$

Thus, $u_{k}(\gamma)$ defines the largest $(i, j)$ (according to the ordering $\xi_{k}$ ) for which $\gamma_{i, j}>0$.

To begin the induction, we set $\tau_{1}=\sigma$ and $\gamma^{*}\left(\tau_{1}\right)=\gamma(\sigma)$, noting that $x_{k}\left(\tau_{1}\right)=\beta_{k}^{\mathrm{e}}\left(\gamma^{*}\left(\tau_{1}\right)\right)$, and initialize the induction variable as $m=1$.

Denote the highest nonempty urn class under the color- $k$ ordering by $u_{k}^{m}=u_{k}\left(\gamma^{*}\left(\tau_{m}\right)\right)$, and denote the corresponding order number by $\kappa_{k}^{m}=\xi_{k}\left(u_{k}^{m}\right)$. Now imagine in reverse time pulling balls from these urns so that mass drains from $\gamma_{u_{1}^{m}}$ at a rate specified by $\dot{x}_{1}$ and from $\gamma_{u_{2}^{m}}$ at a rate specified by $\dot{x}_{2}$. At some time $\tau_{m+1}<\tau_{m}$, one of the two urn classes will empty. If $u_{1}^{m}=u_{2}^{m}$ then this time is given by

$$
\tau_{m+1}=\max \left\{t: \sum_{k=1}^{2} x_{k}\left(\tau_{m}\right)-x_{k}(t)=\gamma_{u_{1}^{1}}^{*}\left(\tau_{m}\right)\right\},
$$


and otherwise it is given by

$$
\tau_{m+1}=\max \left\{t: x_{1}\left(\tau_{m}\right)-x_{1}(t)=\gamma_{u_{1}^{m}}^{*}\left(\tau_{m}\right) \text { or } x_{2}\left(\tau_{m}\right)-x_{2}(t)=\gamma_{u_{2}^{m}}^{*}\left(\tau_{m}\right)\right\} .
$$

Suppose that $u_{1}^{m}=(i, j)$ for some $i>0$. Then we obtain (see (14))

$$
x_{1}\left(\tau_{m}\right)=\beta_{1}^{\mathrm{e}}\left(\gamma^{*}\left(\tau_{m}\right)\right) \geq \gamma_{u_{1}^{m}}^{*}\left(\tau_{m}\right)
$$

so that there must be a solution $t \in\left[0, \tau_{m}\right)$ to $x_{1}(t)=x_{1}\left(\tau_{m}\right)-\gamma_{u_{1}^{m}}^{*}\left(\tau_{m}\right)$. Together with a similar analysis of $u_{2}^{m}$, this means that a solution $\tau_{m+1} \in\left[0, \tau_{m}\right)$ always exists unless $u_{1}^{m}=u_{2}^{m}=(0,0)$. In the latter case, all urns are empty at time $\tau_{m}$, and $\kappa_{1}^{m}+\kappa_{2}^{m}=0$.

Otherwise, if $\kappa_{1}^{m}+\kappa_{2}^{m}>0$, we define

$$
\theta^{1, *}(t)=e_{u_{1}^{m}-(1,0)}, \quad \theta^{2, *}(t)=e_{u_{2}^{m}-(0,1)}
$$

for all $t \in\left[\tau_{m+1}, \tau_{m}\right)$, where $e_{(i, j)}$ is the distribution in $\mathcal{M}$ which puts all mass on the $(i, j)$ th element. That is, during the $m$ th interval, in forward time, we are throwing balls into urns with occupancies $u_{1}^{m}-(1,0)$ and $u_{2}^{m}-(0,1)$ in order to fill the urn classes $u_{k}^{m}$. The process $\gamma^{*}$ is then defined on the interval $\left[\tau_{m+1}, \tau_{m}\right)$ by the existing terminal condition $\gamma^{*}\left(\tau_{m}\right)$ and the differential equation $\dot{\gamma}^{*}=\sum_{k} \dot{x}_{k} T^{k}\left[\theta^{k, *}\right]$. Because the $\theta^{k, *}$ put all their mass on urn classes with $i \leq I$ and $j \leq J$, relations (15) establish that $x\left(\tau_{m+1}\right)=\beta_{k}^{\mathrm{e}}\left(\gamma^{*}\left(\tau_{m+1}\right)\right)$, setting up the next induction step.

Note that $\tau_{m+1}$ was chosen so that at least one of the classes $u_{k}^{m}$ is empty at time $\tau_{m+1}$. This ensures the strict inequality $\sum_{k=1}^{2} \kappa_{k}^{m+1}<\sum_{k=1}^{2} \kappa_{k}^{m}$, so that the induction must terminate after a finite number of steps ( say $M$ ) with $\kappa_{1}^{M}+\kappa_{2}^{M}=0$, meaning that $\gamma^{*}\left(\tau_{M}\right)=e_{(0,0)}=\gamma(0)$. Moreover, the fact that $x_{k}\left(\tau_{M}\right)=\beta_{k}^{\mathrm{e}}\left(\gamma^{*}\left(\tau_{M}\right)\right)=0$ shows that this occurs at time $\tau_{M}=0$.

This establishes the existence of $\gamma^{*}$ and $\theta^{*}$ on $[0, \sigma)$ with $\gamma^{*}(\sigma)=\gamma(\sigma)$ and with $\theta^{*}$ consisting of pure, piecewise-constant controls. In addition, $\gamma_{i, j}^{*}(t)$ increases during intervals when $u_{1}^{m}=(i-1, j)$ or $u_{2}^{m}=(i, j-1)$, and it decreases when $u_{k}^{m}=(i, j)$ for $k=1,2$. By construction, the order numbers $\kappa_{k}^{m}$ increase monotonically with decreasing $m$, ensuring that the intervals on which $\gamma_{i, j}^{*}$ increases precede the intervals of decrease.

Finally, we extend the argument to the case when $x_{k}(\sigma)>\beta_{k}^{\mathrm{e}}(\gamma(\sigma))$ for some $k \in 1,2$. Let $s_{k}$ be the last time in $[0, \sigma]$ such that $x_{k}\left(s_{k}\right)=\beta_{k}^{\mathrm{e}}(\gamma(\sigma))$, and assume without loss of generality that $0<s_{2}<s_{1}<\sigma$. By virtue of (15), the event $x_{1}(t)>\beta_{1}^{\mathrm{e}}(\gamma(t))$ can only occur if balls of class 1 have been thrown into $(I+, j)$-occupied urns, which can only happen with finite cost if these urns hold some mass, i.e. $\sum_{j=0}^{J+} \gamma_{I+, j}(t)>0$.

On the interval $\left[s_{1}, \sigma\right)$, we may define $\theta^{1, *}=e_{(I+, 0)}$ and $\theta^{2, *}=e_{(0, J+)}$. Throwing balls into such urns has no effect on the occupancy distribution, meaning that $\gamma^{*}(t)=\gamma(\sigma)$ is the solution on this interval to the equation

$$
\dot{\gamma}^{*}=\sum_{k=1}^{2} \dot{x}_{k} T^{k}\left[\theta^{k, *}\right]=0 .
$$

The desired property that $\theta_{i, j}(t)>0$ implies $\gamma_{i, j}(t) \geq \delta \sigma^{k}$ is trivially satisfied on this interval.

At time $s_{1}$, we have $x_{1}\left(s_{1}\right)=\beta_{1}^{\mathrm{e}}\left(\gamma^{*}\left(s_{1}\right)\right)$ and $x_{2}\left(s_{1}\right)>\beta_{2}^{\mathrm{e}}\left(\gamma^{*}\left(s_{1}\right)\right)$. On the interval $\left[s_{2}, s_{1}\right)$, we use a modified reverse-time induction in which

$$
\tau_{m+1}=\max \left\{t: x_{1}\left(\tau_{m}\right)-x_{1}(t)=\gamma_{u_{1}^{m}}^{*}\left(\tau_{m}\right) \text { or } t=s_{2}\right\} .
$$


On the $m$ th subinterval, the occupancy rates naturally are defined as $\theta^{1, *}=e_{u_{1}^{m}-(1,0)}$ and $\theta^{2, *}=e_{(0, J+)}$. Using similar arguments to before, it follows that, after a finite number of steps, the induction terminates with $\tau_{M}=s_{2}, \gamma_{0, J+}^{*}\left(s_{2}\right) \geq \gamma_{0, J+}(\sigma)$, and $\kappa_{1}^{m}$ strictly decreasing in $m$. Since now $x_{k}\left(s_{2}\right)=\beta_{k}^{\mathrm{e}}\left(\gamma^{*}\left(s_{2}\right)\right), k=1,2$, the original induction argument may be used to continue the definition of $\gamma^{*}$ on $\left[0, s_{2}\right)$. Now both $\kappa_{1}^{m}$ and $\kappa_{2}^{m}$ are both strictly decreasing in $m$ across the entire interval $[0, \sigma)$, and we have the desired property that $\theta_{i, j}>0$ implies $\gamma_{i, j}^{*}(s) \geq \gamma_{i, j}(\sigma)$ for $s \in[0, \sigma]$.

This completes the construction of the processes $\gamma^{*}, \theta^{1, *}$, and $\theta^{2, *}$ on $[0, \sigma]$. Note that

$$
\begin{aligned}
\int_{0}^{\sigma}\left[\dot{x}_{1} R\left(\theta^{1, *} \| \gamma^{*}\right)+\dot{x}_{2} R\left(\theta^{2, *} \| \gamma^{*}\right)\right] \mathrm{d} s & \leq-\int_{0}^{\sigma}\left[\dot{x}_{1} \log \left(\delta \sigma^{K}\right)+\dot{x}_{2} \log \left(\delta \sigma^{K}\right)\right] \mathrm{d} s \\
& =-\sigma \log \left(\delta \sigma^{K}\right) \\
& \leq \frac{\varepsilon}{2}
\end{aligned}
$$

and that $\gamma^{*}$ deviates by no more than $\varepsilon$ from $\gamma$ on $[0, \sigma]$, while ending up at the same place, i.e. $\gamma^{*}(\sigma)=\gamma(\sigma)$.

The construction on $[\sigma, \tau]$ is simpler. Let $M \in \mathbb{N}$, and observe that $\gamma_{i, j}(s)$ is uniformly bounded away from zero for all $i$ and $j$ and $s \in[\sigma, \tau]$. We partition $[\sigma, \tau]$ into $M$ subintervals of length $c_{M}=(\tau-\sigma) / M$. On each interval we set

$$
\theta_{i, j}^{k, *}(s)=\frac{\int_{\sigma+l c_{M}}^{\sigma+(l+1) c_{M}} \dot{x}_{k}(r) \theta_{i, j}^{k}(r) \mathrm{d} r}{x_{k}\left(\sigma+(l+1) c_{M}\right)-x_{k}\left(\sigma+l c_{M}\right)}
$$

if $\sigma+l c_{M} \leq s \leq \sigma+(l+1) c_{M}$ (the definition is unimportant if $x_{k}\left(\sigma+(l+1) c_{M}\right)-$ $\left.x_{k}\left(\sigma+l c_{M}\right)=0\right)$. For $s \in[\sigma, \tau]$, let

$$
\dot{\gamma}^{*}=\dot{x}_{1} T^{1}\left[\theta^{1, *}\right]+\dot{x}_{2} T^{2}\left[\theta^{2, *}\right], \quad \gamma^{*}(\sigma)=\gamma(\sigma) .
$$

Since $\gamma_{i, j}(s)$ is uniformly bounded away from zero, it is easy to check that, for large enough $M, \gamma^{*}$ is a valid occupancy path that is associated with the processes $\theta^{1, *}, \theta^{2, *}$, and $x$. The convergence $\gamma^{*} \rightarrow \gamma$ on $[\sigma, \tau]$ as $M \rightarrow \infty$ is immediate, and it follows from the Lebesgue dominated convergence theorem that

$$
\lim _{M \rightarrow \infty} \int_{\sigma}^{\tau}\left[\dot{x}_{1} R\left(\theta^{1, *} \| \gamma^{*}\right)+\dot{x}_{2} R\left(\theta^{2, *} \| \gamma^{*}\right)\right] \mathrm{d} s=\int_{\sigma}^{\tau}\left[\dot{x}_{1} R\left(\theta^{1} \| \gamma\right)+\dot{x}_{2} R\left(\theta^{2} \| \gamma\right)\right] \mathrm{d} s .
$$

Therefore, all parts of the lemma hold for sufficiently large $M$.

\section{Proof of the large deviation lower bound}

In the proof of the lower bound it will be sufficient to work with the occupancy paths with the properties set out in Lemma 6. Given such a path $\gamma$, the overall idea of the proof is to construct a sequence of measures with sample paths close to $\gamma$ in distance and with nearly equal costs. We first show that we may concentrate on coloring processes lying within some small distance from those obtaining the infimum for $\gamma$. Furthermore, urn selection is uniform on each type $(i, j)$ with mass equal to the (piecewise-constant) derivatives of $\gamma$. Finally, by using a stopping time argument, we show that the sample path under the above measure lies arbitrarily close to $\gamma$ with probability approaching 1 . Estimates of the cost are readily constructed due to lower bounds on the components of $\gamma$ and because the derivatives of the paths in Lemma 6 are piecewise constant. 
Theorem 4. Under Assumption 2, we have

$$
\limsup _{n \rightarrow \infty}\left[-\frac{1}{n} \log \mathrm{E} \exp \left[-n F\left(\Gamma^{n}\right)\right]\right] \leq \inf _{\gamma \in \mathcal{S}}[I(\gamma)+F(\gamma)] .
$$

Proof. Consider any $\gamma$ for which $I(\gamma)<\infty$. Then it suffices to show that

$$
\limsup _{n \rightarrow \infty}\left[-\frac{1}{n} \log \mathrm{E} \exp \left[-n F\left(\Gamma^{n}\right)\right]\right] \leq I(\gamma)+F(\gamma) .
$$

Owing to Lemma 5 and the continuity of $F$, we can assume without loss of generality that there exist $\delta>0$ and $K \in \mathbb{N}$ such that $\gamma_{i, j}(t) \geq \delta t^{K}$.

We again utilize representation (2). Fix $b>0$. According to the representation, inequality (16) will follow if we can find a sequence $\left\{\mu^{n}, n \in \mathbb{N}\right\}$ such that

$$
\limsup _{n \rightarrow \infty} \frac{1}{n} R\left(\mu^{n} \| \Pi^{n} \otimes \Lambda^{n}\right) \leq I(\gamma)+b,
$$

and such that if $\bar{\Gamma}^{n}$ is the urn process constructed under the distribution $\mu^{n}$, then

$$
\limsup _{n \rightarrow \infty} \overline{\mathrm{P}}^{n}\left\{d\left(\bar{\Gamma}^{n}, \gamma\right)>b\right\} \leq b .
$$

To prove the desired bound we must construct an appropriate sequence of measures $\mu^{n}$. For $\gamma$ as above, let $\theta^{1}, \theta^{2}$, and $\tilde{x}$ denote the corresponding rate and coloration processes which achieve the infimum. Without loss of generality we can assume that these processes satisfy the properties ascribed to $\theta^{1, *}$ and $\theta^{2, *}$ in Lemma 6 .

For $a>0$, let $G: \mathcal{T} \rightarrow \mathbb{R}$ be continuous and satisfy

$$
G(x)= \begin{cases}\frac{1}{a} & \text { if } d(x, \tilde{x}) \geq 2 a, \\ 0 & \text { if } d(x, \tilde{x}) \leq a\end{cases}
$$

and also $G(x) \in[0,1 / a]$ for all $x \in \mathcal{T}$. Since $\left\{X^{n}, n \in \mathbb{N}\right\}$ satisfies a large deviations principle with rate function $J$, there exists a sequence $\left\{\lambda^{n}, n \in \mathbb{N}\right\}$, satisfying

$$
\begin{aligned}
\overline{\mathrm{E}}^{n}\left[\frac{1}{n} R\left(\lambda^{n} \| \Lambda^{n}\right)+G\left(\bar{X}^{n}\right)\right] & \rightarrow \inf _{x \in \mathcal{T}}[J(x)+G(x)] \\
& \leq J(\tilde{x}) .
\end{aligned}
$$

We use these measures in constructing $\mu^{n}$ by setting

$$
\begin{aligned}
& \mu^{n}\left(\mathrm{~d} u_{1}, \ldots, \mathrm{d} u_{N_{n}}, \mathrm{~d} y_{1}, \ldots, \mathrm{d} y_{N_{n}}\right) \\
&= \lambda^{n}\left(\mathrm{~d} y_{1}, \ldots, \mathrm{d} y_{N_{n}}\right) \\
& \times \mu_{1}^{n}\left(\mathrm{~d} u_{1} \mid y_{1}, \ldots, y_{N_{n}}\right) \cdots \mu_{N_{n}}^{n}\left(\mathrm{~d} u_{N_{n}} \mid u_{1}, \ldots, u_{N_{n}-1}, y_{1}, \ldots, y_{N_{n}}\right) .
\end{aligned}
$$

We will need to know how much $\lambda^{n}$ mass is placed on sequences $y_{1}, \ldots, y_{\lfloor n \tau\rfloor+1}$, such that if $\bar{X}^{n}$ is the corresponding cumulative coloration process then

$$
\sup _{t \in[0, \tau]} d\left(\bar{X}^{n}(t), \tilde{x}(t)\right) \geq 2 a .
$$


We have

$$
\begin{aligned}
\limsup _{n \rightarrow \infty} \frac{1}{a} \overline{\mathrm{P}}^{n}\left\{\sup _{t \in[0, \tau]} d\left(\bar{X}^{n}(t), \tilde{x}(t)\right) \geq 2 a\right\} & \leq \limsup _{n \rightarrow \infty} \overline{\mathrm{E}}^{n}\left[G\left(\bar{X}^{n}\right)\right] \\
& \leq \inf _{x \in \mathcal{T}}[J(x)+G(x)] \\
& \leq J(\tilde{x}) \\
& <\infty .
\end{aligned}
$$

Thus, the probability $\overline{\mathrm{P}}^{n}\left\{\sup _{t \in[0, \tau]} d\left(\bar{X}^{n}(t), \tilde{x}(t)\right) \geq 2 a\right\}$ can be made as small as desired for large $n$ by taking $a$ small. Since $\|F\|_{\infty}<\infty$, when constructing the controlled urn process we will be able to ignore these paths, and can let the measures that select the urns be the original uniform measure for such points in the underlying probability space. The relative entropy cost for such paths is then zero. Thus, in the rest of this construction we focus on the case where the underlying coloration process satisfies

$$
\sup _{t \in[0, \tau]} d\left(\bar{X}^{n}(t), \tilde{x}(t)\right) \leq 2 a .
$$

To finish the construction we must specify the conditional distributions of the $U_{l}^{n}$. Note that when specifying these distributions we get to see the complete outcome $Y_{1}^{n}, \ldots, Y_{N_{n}}^{n}$ of the coloration process, and can assume that this process satisfies (19).

The construction naturally separates according to the partition $[0, \tau]=[0, \sigma) \cup[\sigma, \tau]$. We must specify the distribution of the measures $\mu_{l}^{n}$ (or, equivalently, the distribution of the random measures $\bar{\mu}_{l}^{n}$ ) for $l=1, \ldots, N_{n}$. Recall that in general these measures are allowed to depend on the 'past' $U_{q}^{n}, q<l$. However, it will turn out that we can assign $\mu_{l}^{n}$ based on just the coloration sequence and the time index $l$ (i.e. 'open-loop' controls). Let $\left(s_{1}^{m}, s_{2}^{m}\right)$ denote the finite collection of intervals on which $\theta_{i, j}^{k}(t)$ is constant, so that these intervals are nonoverlapping, and $[0, \tau] \backslash \bigcup_{m=1}^{M}\left(s_{1}^{m}, s_{2}^{m}\right)$ consists of a finite number of points. Suppose that $l / n \in\left[s_{1}^{m}, s_{2}^{m}\right)$.

- If $s_{2}^{m} \leq \sigma$ then for each $k$ there exists $(i, j)$ such that $\theta_{i, j}^{k}(t)=1$ for $t \in\left(s_{1}^{m}, s_{2}^{m}\right)$. If $Y_{l}^{n}=k$ (the ball at time $l$ is color $k$ ) then $\mu_{l}^{n}$ is set to be the uniform distribution on all urns of class $(i, j)$. Note that when we rewrite the relative entropy as in (5) there will be equality, and in fact

$$
\begin{aligned}
\overline{\mathrm{E}}^{n}\left[R\left(\bar{\mu}_{l}^{n} \| \pi^{n}\right)\right] & =\overline{\mathrm{E}}^{n}\left[R\left(v^{n}\left(\frac{l}{n}\right) \| \bar{\Gamma}^{n}\left(\frac{l}{n}\right)\right)\right] \\
& =\overline{\mathrm{E}}^{n}\left[\sum_{i=0, j=0}^{I+, J+} \log \left(\frac{v_{i, j}^{n}(l / n)}{\bar{\Gamma}_{i, j}^{n}(l / n)}\right) v_{i, j}^{n}\left(\frac{l}{n}\right)\right] \\
& =-\overline{\mathrm{E}}^{n} \log \left(\bar{\Gamma}_{i, j}^{n}\left(\frac{l}{n}\right)\right) .
\end{aligned}
$$

- If $s_{2}^{m}>\sigma$ then the controls are no longer 'pure'. If $y_{l}=k$ then each $\theta_{i, j}^{k}(t)$ determines a 'weight' that should be placed on urns of class $(i, j)$. We let $\mu_{l}^{n}$ be the measure which places mass $\theta_{i, j}^{k}(t)$ on the urns of class $(i, j)$, and within this class uses the uniform distribution to apportion mass. We again have equality in the relative entropy in (5), and 
in fact

$$
\begin{aligned}
\overline{\mathrm{E}}^{n}\left[R\left(\bar{\mu}_{l}^{n} \| \pi^{n}\right)\right] & =\overline{\mathrm{E}}^{n}\left[R\left(v^{n}\left(\frac{l}{n}\right) \| \bar{\Gamma}^{n}\left(\frac{l}{n}\right)\right)\right] \\
& =\overline{\mathrm{E}}^{n}\left[\sum_{i=0, j=0}^{I+, J+} \log \left(\frac{\theta_{i, j}^{k}(l / n)}{\bar{\Gamma}_{i, j}^{n}(l / n)}\right) \theta_{i, j}^{k}\left(\frac{l}{n}\right)\right] .
\end{aligned}
$$

Note that for the controls constructed in Lemma 6 we have $\gamma_{i, j}(t) \geq \delta \sigma^{K}$ for any $(i, j)$ for which $\theta_{i, j}^{1}(t) \vee \theta_{i, j}^{2}(t)>0$. Owing to the randomness of the prelimit processes, we cannot guarantee the corresponding result $\bar{\Gamma}_{i, j}^{n}(t) \geq \delta \sigma^{K}$ for any $(i, j)$ for which $\theta_{i, j}^{1}(t) \vee \theta_{i, j}^{2}(t)>0$. We therefore use a stopping time argument in the construction of the measures $\mu_{l}^{n}$. Let $\bar{l}_{n}$ be the first time $l$ such that $\bar{\Gamma}_{i, j}^{n}(l / n) \leq \delta \sigma^{K} / 2$, for some $(i, j)$ for which $\theta_{i, j}^{1}(l / n) \vee \theta_{i, j}^{2}(l / n)>0$. From time $\bar{l}_{n}$ on, the construction above is modified, in that the measure is selected so that $v^{n}(l / n)=\bar{\Gamma}^{n}(l / n)$ for $l \geq \bar{l}_{n}$. Thus, a weight of $\bar{\Gamma}_{i, j}^{n}(l / n)$ is placed on the urns of class $(i, j)$. Note that with this definition $\overline{\mathrm{E}}^{n}\left[R\left(v^{n}(l / n) \| \bar{\Gamma}^{n}(l / n)\right)\right]=0$ for $l \geq \bar{l}_{n}$. We now apply Theorem 1. Thus, given any subsequence we have convergence along a further subsequence as indicated in the theorem, with limit $\left(\bar{\Gamma}, \bar{X}_{1}, \bar{X}_{2}, \bar{\theta}^{1}, \bar{\theta}^{2}\right)$. Using the standard argument by contradiction, it will be enough to prove the convergence of controlled processes and bounds on the relative entropy cost for this convergent subsequence. Let $\rho^{n}=\left(\bar{l}_{n} / n\right) \wedge \tau$. Note that because the applied controls are pure the process $\bar{\Gamma}^{n}(t)$ is deterministic prior to $\sigma$, and also that prior to this time the time derivatives of both $\bar{\Gamma}^{n}(t)$ and $\gamma(t)$ are piecewise constant. In fact, the two derivatives are identical except possibly on a bounded number of intervals of length less than $1 / n$ (located near the endpoints of the intervals of constancy of $\dot{\gamma}(t)$ ). Thus, for large $n$ we cannot have $\rho^{n}<\sigma$. Since the range of $\rho^{n}$ is bounded, we can also assume that $\rho^{n}$ converges (along the same subsequence) in distribution to a limit $\rho$, and it is easy to check that the limit control processes almost everywhere satisfy

$$
\bar{\theta}_{i, j}^{k}(t)= \begin{cases}\theta_{i, j}^{k}(t) & \text { if } t \leq \rho, \\ \bar{\Gamma}_{i, j}(t) & \text { if } t>\rho .\end{cases}
$$

Owing to the definition of $\rho^{n}$, if $\rho<\tau$ then $\bar{\Gamma}_{i, j}(\rho)=\delta \sigma^{K} / 2$ for some $(i, j)$. Recall also that $\gamma_{i, j}(t) \geq \delta \sigma^{K}$ for all $t \in[\sigma, \tau]$.

Observe that the limit processes all implicitly depend on $a>0$ through the function $G$. We claim that, for each $b>0$,

$$
\lim _{a \downarrow 0} \overline{\mathrm{P}}\{d(\bar{\Gamma}, \gamma)>b\}=0 .
$$

We already know that

$$
\lim _{a \downarrow 0} \overline{\mathrm{P}}\{d(\bar{X}, \tilde{x})>2 a\}=0 .
$$

However, since the rate processes $\theta_{i, j}^{k}$ are all piecewise constant, the integral

$$
\int_{0}^{t}\left(\dot{\bar{X}}_{1} T^{1}\left[\theta^{1}\right]+\dot{\bar{X}}_{2} T^{2}\left[\theta^{2}\right]\right) d s
$$

converges uniformly to

$$
\int_{0}^{t}\left(\dot{\tilde{x}}_{1} T^{1}\left[\theta^{1}\right]+\dot{\tilde{x}}_{2} T^{2}\left[\theta^{2}\right]\right) \mathrm{d} s
$$


as $d(\bar{X}, \tilde{x}) \rightarrow 0$. Therefore,

$$
\lim _{a \downarrow 0} \overline{\mathrm{P}}\left\{\sup _{0 \leq t \leq \rho}\|\bar{\Gamma}(t)-\gamma(t)\|>b\right\}=0 .
$$

If $b>0$ is sufficiently small, then the following three items, all of which hold under $\rho<\tau$, form a contradiction:

- $\bar{\Gamma}_{i, j}(\rho)=\delta \sigma^{K} / 2$ for some $(i, j)$,

- $\gamma_{i, j}(t) \geq \delta \sigma^{K}$ for all $t \in[0, \tau]$,

- $\sup _{0 \leq t \leq \rho}\|\bar{\Gamma}(t)-\gamma(t)\| \leq b$.

We conclude that $\lim _{a \downarrow 0} \overline{\mathrm{P}}\{\rho<\tau\}=0$ and, therefore, $\lim _{a \downarrow 0} \overline{\mathrm{P}}\{d(\bar{\Gamma}, \gamma)>b\}=0$ for all sufficiently small $b>0$. It follows that, given $b>0$, for some fixed (sufficiently small) $a>0$, $\lim \sup _{n \rightarrow \infty} \overline{\mathrm{P}}\left\{d\left(\bar{\Gamma}^{n}, \gamma\right)>b\right\} \leq b$.

We must also consider the relative entropy costs. However, again using the convergence $\lim _{a \downarrow 0} \overline{\mathrm{P}}\{d(\bar{\Gamma}, \gamma)>b\}=0$ and the dominated convergence theorem,

$$
\begin{aligned}
& \underset{a \downarrow 0}{\lim \sup } \limsup _{n \rightarrow \infty} \overline{\mathrm{E}}^{n}\left[\frac{1}{n} \sum_{l=1}^{N_{n}} R\left(v^{n}\left(\frac{l}{n}\right) \| \bar{\Gamma}^{n}\left(\frac{l}{n}\right)\right)\right] \\
& =\underset{a \downarrow 0}{\lim \sup } \limsup _{n \rightarrow \infty} \overline{\mathrm{E}}^{n}\left[\sum _ { m = 1 } ^ { M } \left(\sum_{l=\left\lfloor n s_{1}^{m}\right\rfloor+1}^{\left\lfloor n s_{2}^{m}\right\rfloor} R\left(\theta^{1}\left(\frac{l}{n}\right) \| \bar{\Gamma}^{n}\left(\frac{l}{n}\right)\right)\right.\right. \\
& \times\left(X_{1}^{n}\left(\frac{l+1}{n}\right)-X_{1}^{n}\left(\frac{l}{n}\right)\right) \\
& +\sum_{l=\left\lfloor n s_{1}^{m}\right\rfloor+1}^{\left\lfloor n s_{2}^{m}\right\rfloor} R\left(\theta^{2}\left(\frac{l}{n}\right) \| \bar{\Gamma}^{n}\left(\frac{l}{n}\right)\right) \\
& \left.\left.\times\left(X_{2}^{n}\left(\frac{l+1}{n}\right)-X_{2}^{n}\left(\frac{l}{n}\right)\right)\right)\right] \\
& =\underset{a \downarrow 0}{\lim \sup } \overline{\mathrm{E}}\left[\sum_{m=1}^{M}\left(\int_{s_{1}^{m}}^{s_{2}^{m}} R\left(\theta^{1}(t) \| \bar{\Gamma}(t)\right) \mathrm{d} \bar{X}_{1}(t)+\int_{s_{1}^{m}}^{s_{2}^{m}} R\left(\theta^{2}(t) \| \bar{\Gamma}(t)\right) \mathrm{d} \bar{X}_{2}(t)\right)\right] \\
& =\int_{0}^{\tau}\left[\dot{\tilde{x}}_{1}(t) R\left(\theta^{1}(t) \| \gamma(t)\right)+\dot{\tilde{x}}_{2}(t) R\left(\theta^{2}(t) \| \gamma(t)\right)\right] \mathrm{d} t .
\end{aligned}
$$

When this is combined with the bound

$$
\limsup _{n \rightarrow \infty} \frac{1}{n} R\left(\lambda^{n} \| \Lambda^{n}\right) \leq J(\tilde{x})
$$

for small enough $a>0$, we have proved (17). Since $\lim _{a \downarrow 0} \overline{\mathrm{P}}\{d(\bar{\Gamma}, \gamma)>b\}=0$ implies (18) for small $a>0$, the proof is complete. 


\section{References}

[1] Bailey, N. T. J. (1951). On estimating the size of mobile populations from recapture data. Biometrika 38, 293-306.

[2] Boucheron, S., Gamboa, F. And Leonard C. (2002). Bins and balls: large deviations of the empirical occupancy process. Ann. Appl. Prob. 2, 1-30.

[3] Chao, A. (2001). An overview of closed capture-recapture models. J. Agricultural Biol. Environ. Statist. 6, $158-175$

[4] Dupuis, P. And Ellis, R. S. (1997). A Weak Convergence Approach to Large Deviations. John Wiley, New York.

[5] Dupuis, P., Ellis, R. S. AND Weiss, A. (1991). Large deviations for Markov processes with discontinuous statistics. I. General upper bounds. Ann. Prob. 19, 1280-1297.

[6] Dupuis, P., Nuzman, C. And Whiting, P. (2003). Occupancy models and circuit switched networks with blocking. In Proc. 41st Annual Allerton Conf. Commun. Control Comput. (September 2003), University of Illinois Press, Champaign, IL.

[7] Dupuis, P., Nuzman, C. And Whiting, P. (2004). Large deviation asymptotics for occupancy problems. Ann. Prob. 32, 2765-2818.

[8] Eramo, V., Listanti, M., Nuzman, C. and Whiting, P. (2002). Optical switch dimensioning and the classical occupancy problem. Internat. J. Commun. 15, 127-141.

[9] Finkelstein, M., Tucker, H. G. And Veeh, J. A. (1998). Confidence intervals for the number of unseen types. Statist. Prob. Lett. 37, 423-430.

[10] Graham, C. and O'Connell, N. (2002). Large deviations at equilibrium for a large star-shaped loss network. Ann. Appl. Prob. 2, 1807-1856.

[11] Johnson, N. L. And Kotz, S. (1977). Urn Models and Their Application. John Wiley, New York.

[12] Kelly, F. P. and Ziedins, I. (1989). Blocking in star networks. Adv. Appl. Prob. 21, 804-830.

[13] Kotz, S. And Balakrishnan, N. (1997). Advances in urn models during the past two decades. In Advances in Combinatorial Methods and Applications to Probability and Statistics, ed. N. Balakrishnan, Birkhäuser, Boston, MA, pp. 203-257.

[14] Seber, G. A. F. (1982). The Estimation of Animal Abundance and Related Parameters, 2nd edn. Macmillan, New York.

[15] VAnder Wiel, S. A. And Votta, L. G. (1993). Assessing software designs using capture-recapture methods. IEEE Trans. Software Eng. 19, 1045-1054.

[16] Whiтt, W. (1985). Blocking when service is required from several facilities simultaneously. AT\&T Tech. J. 64, $1807-1856$ 